\title{
Solving fuzzy multi-objective shortest path problem based on data envelopment analysis approach
}

\author{
M. Bagheri ${ }^{1}$ - Ali Ebrahimnejad ${ }^{2}$ S. Razavyan ${ }^{1} \cdot$ F. Hosseinzadeh Lotf ${ }^{3} \cdot$ N. Malekmohammadi ${ }^{1}$
}

Received: 13 July 2020 / Accepted: 12 November 2020 / Published online: 2 January 2021

(c) The Author(s) 2021

\begin{abstract}
The shortest path problem (SPP) is a special network structured linear programming problem that appears in a wide range of applications. Classical SPPs consider only one objective in the networks while some or all of the multiple, conflicting and incommensurate objectives such as optimization of cost, profit, time, distance, risk, and quality of service may arise together in real-world applications. These types of SPPs are known as the multi-objective shortest path problem (MOSPP) and can be solved with the existing various approaches. This paper develops a Data Envelopment Analysis (DEA)-based approach to solve the MOSPP with fuzzy parameters (FMOSPP) to account for real situations where input-output data include uncertainty of triangular membership form. This approach to make a connection between the MOSPP and DEA is more flexible to deal with real practical applications. To this end, each arc in a FMOSPP is considered as a decision-making unit with multiple fuzzy inputs and outputs. Then two fuzzy efficiency scores are obtained corresponding to each arc. These fuzzy efficiency scores are combined to define a unique fuzzy relative efficiency. Hence, the FMOSPP is converted into a single objective Fuzzy Shortest Path Problem (FSPP) that can be solved using existing FSPP algorithms.
\end{abstract}

Keywords Shortest path problem · Fuzzy numbers · Data envelopment analysis · Fuzzy efficiency

Mathematics Subject Classification 90B06 $\cdot 90 \mathrm{C} 70$

\section{Introduction}

Network optimization models can be seen in a wide range of application such as transportation systems, communication systems, pipeline distribution systems, fluid flow systems and neural decision systems [1]. In many real-life applications, it is required to obtain a best or shortest paths from one node to another. This kind of problem as one of the important types of network models is called shortest path problem (SPP). However, the best path cannot be mostly measured by a single objective alone, but by some or all of them together. In such cases, we will encounter a multi-objective shortest

Ali Ebrahimnejad

aemarzoun@gmail.com; a.ebrahimnejad@qaemiau.ac.ir

1 Department of Mathematics, South Tehran Branch, Islamic Azad University, Tehran, Iran

2 Department of Mathematics, Qaemshahr Branch, Islamic Azad University, Qaemshahr, Iran

3 Department of Mathematics, Science and Research Branch, Islamic Azad University, Tehran, Iran path problem (MOSPP). In these problems, multiple, conflicting and incommensurate objectives such as optimization of cost, profit, time, distance, risk, quality of service etc. may arise. There are various approaches to solve MOSPPs. For example, Martins and Santos [2] analyzed the labeling algorithm for the MOSPP by the generalization of the classical shortest path problem. Sastry et al. [1] proposed three algorithms for MOSPP and an algorithm to detect negative cycle in a network. Using their proposed algorithms, MOSPP in a cyclic and acyclic network having weights either positive or negative or both can be solved. Sauvanet and Neron [3] focused on the determination of a well-balanced trade-off path between the objectives. They recalled the original Best Compromise $\mathrm{A}^{*}$ method and then presented two improvements to speed up the search of the best compromise solution. Pulido et al. [4] introduced an exact label-setting algorithm that returns the subset of Pareto optimal paths that satisfy a set of lexicographic goals, or the subset that minimizes deviation from goals if these cannot be fully satisfied. Shi et al. [5] proposed an exact method for finding all the Paretooptimal paths for a multi-criteria constrained shortest path

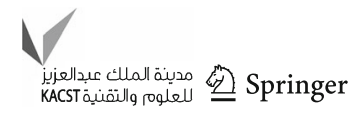


problem. Cintrano et al. [6] proposed a robust model for the bi-objective shortest path problem applied in a smart mobility context. Zajac and Huber [7] proposed a classification scheme in which every identified objective (in a given multi-objective routing problem) is sorted into a category and related to problem elements. Ajeil et al. [8] solved a path planning problem for an autonomous mobile robot in static and dynamic environments. Dehghani et al. [9] proposed a multi-objective optimization model for reliable communication flow networks, including maximizing the network reliability, minimizing total cost, and maximizing network flow, simultaneously.

Among the aforementioned methods, there are a number that are based on data envelopment analysis (DEA). DEA is a widely used technique to evaluate the performance of a set of homogeneous decision making units (DMUs). Nowadays, extensive researches have been performed in DEA and its scope has been broadened. "One reason is that DEA has opened up possibilities for use in cases which have been resistant to other approaches because of the complex (often unknown) nature of the relations between the multiple inputs and multiple outputs involved in many of these activities" [10]. The DEA-based approaches to make a connection between the MOSPP and efficiency are more flexible to deal with the real-world applications. Among these approaches, the work of Masoumi et al. [11] can be mentioned that considered each possible path from the source node to the destination one as a DMU. They proved that each nondominated path in the MOSPP is equivalent to the strongly efficient DMU under the Variable Returns to Scale (VRS) FDH model, and in this way, they tried to determine the set of all non-dominated paths on the network. Amirteimoori et al. [12] obtained a unique efficiency measure corresponding to each arc using DEA models. Then he defined the overall efficiency for each possible path and determined an efficient path, a path with maximum overall efficiency, as an optimal solution to the MOSPP.

In this paper, we develop a DEA-based approach to solve the MOSPP with fuzzy parameters. As you know, for various reasons such as shortage of information, insufficient data, lack of evidence, and so forth, the decision maker usually does not have exact information about the parameters in the real-life applications of MOSPP. In fact, in classical MOSPP it is assumed that the costs and time of paths are exactly known. In real-life shortest path cases, decision-makers may face many uncertainties on the cost and time of paths because of aesthetic quality of the scenery and changing weather, social, or economic conditions. In other words, the decisionmakers cannot exactly know the parameters of a MOSPP in reality. Additionally, these parameters are not stable since this imprecision may follow from the lack of exact information or data, uncertainty in judgement and high information cost. This imprecision embedded into the parameters may not be of a probabilistic type, so this type of uncertainty can be handled via fuzzy parameters. The MOSPP with at least one fuzzy parameter is called Fuzzy MOSPP (FMOSPP). There are few methods to solve the FMOSPPs. For example, Rani and Reddy [13] discussed the multi-objective fuzzy shortest path selection, where the arc lengths are expressed as trapezoidal fuzzy numbers. Zero et al. [14] proposed two algorithms to the Bi-objective Shortest Path (BSP) problem considering a fuzzy objective. Hasuike [15] proposed a model for a robust shortest path problem with random arc costs. The author formulated the proposed model as a bi-criteria optimization problem to minimize the total cost of the path from a source node to a destination node as well as to maximize the range of the confidence interval satisfying the condition that the total cost of any path in the region is less than a target value in terms of robustness. Patle et al. [16] presented the efficient obstacle avoidance mechanism in a complex environment based on the fuzzy optimized decision function.

Abbaszdeh Sori et al. [17] proposed an approach using fuzzy inference system to find the most desirable path of a fuzzy constrained SSP based on three factors of cost, time, and risk which the length of path does not exceed a predetermined value. Majumder et al. [18] presented an uncertain MOSPP for a weighted connected directed graph, where every arc weight is associated with uncertain cost and time. They have formulated the expected value model and chanceconstrained model of the proposed uncertain MOSPP and employed two multi-objective genetic algorithms for solving these models. Nonetheless, to the best of our knowledge, there is very limited literature available based on fuzzy DEA to solve the FMOSPPs.

Note that each arc in a FMOSPP corresponds to multiple fuzzy attributes. In this paper, using Fuzzy DEA (FDEA) models, each batch of multiple fuzzy attributes associated with the arcs is converted into a unique fuzzy attribute. To do this, each arc in a FMOSPP is considered as a DMU with multiple fuzzy inputs and outputs. Indeed, the objective functions that need to be maximized are used to define outputs and those ones that need to be minimized are used to define inputs. Then two fuzzy efficiency scores are obtained corresponding to each arc. These fuzzy efficiency scores are combined to define a unique fuzzy attribute. By considering the unique fuzzy relative efficiency as the unique attribute of the arcs, the FMOSPP under consideration is converted into a single objective fuzzy shortest path problem (FSPP) that can be solved using the existing algorithms for solving fuzzy FSPPs such as the generalized Dijkstra algorithm proposed by Deng et al. [19]. The artificial bee colony (ABC) algorithm proposed by Ebrahimnejad et al. [20] can also be used to solve the fuzzy SP (FSP) problems with fuzzy arc weights. The proposed algorithm by Tajdin et al. [21] can compute a shortest path in a network having various types of fuzzy arc lengths. Moreover, the fuzzy-based modified particle swarm 
optimization algorithm [22] and elite artificial bees' colony algorithm [23] can be used for solving FSP problem. An overview of efficient algorithms for solving SP problems with various types of input data in junction with fuzzy, intuitionistic, vague, interval fuzzy, interval-valued intuitionistic fuzzy and neutrosophic sets can be found in Broumi et al. [24].

It should be mentioned that there are various FDEA methods to measure the efficiency of DMUs with fuzzy inputs and outputs. For example, the tolerance approach developed by Sengupta [25] is one of the first fuzzy DEA models. Chen [26] modified the $\alpha$-level approach and proposed an alternative fuzzy DEA to handle both the crisp and fuzzy data. Saati and Memariani [27] developed a fuzzy slack-based measure (SBM) based on the $\alpha$-level approach. Hatami-Marbini et al. [28] used the provided ranking method in Asady and Zendehnam [29] to propose a fuzzy DEA model for assessing the efficiency scores in the fuzzy environment. Sheth and Triantis [30] introduced a fuzzy goal DEA framework to measure and evaluate the goals of efficiency and effectiveness in a fuzzy environment. Azar et al. [31] developed a new model for calculating the common set of weights (CSW) in fuzzy environment. Ebrahimi et al. [32] proposed a pair of DEA models to find the lower and upper bound efficiency scores in the presence of interval and weak ordinal data. Heydari et al. [33] presented a fully fuzzy network DEARange Adjusted Measure model for evaluating airlines and extends the network DEA-RAM model in the fully fuzzy framework. Arana-Jimenez et al. [34] proposed slacks-based additive inefficiency measure to deal with the problem of efficiency assessment when the input and output data are given as fuzzy sets. Another method that underpins this paper is the work of Saati et al. [35]. They presented a fuzzy version of CCR model [36] with the asymmetrical triangular fuzzy number and suggested a procedure for its solution. The basic idea in this procedure is to transform the fuzzy CCR model into a crisp linear programming problem by applying an alternative $\alpha$-cut approach. In this way, the procedure converts the problem to an interval programming in which, instead of comparing the equality (or inequality) of two intervals, a variable is defined in the interval, not only satisfies the set of constraints but also maximizes the efficiency value.

On such motivation basis, the main contributions of this study are summarized as follows: (1) To the best of our knowledge, this study is the first attempt for solving FMOSPP using FDEA approach, (2) The use of the FDEA approach allows the simultaneous use of maximization and minimization functions in modeling the FMOSPP, (3) The proposed FDEA approach gives an efficient plan in converting FMOSPP into a crisp single objective SPP, (4) In contrast to the existing multi objective optimization solution approaches such as goal programming, the proposed solution technique keeps the structure of SPP for ease of solution and implementation, (5) The FDEA approach proposed in this paper provided an efficient plan for the FMOSPP without explicit the knowledge of the decision maker's fuzzy utility function.

The rest of the paper is organized as follows: Section 2 presents the necessary mathematical background including some basic definitions about the fuzzy numbers along with the preliminaries about the FDEA. A brief introduction to the FMOSPP is presented in Sect. 3. In Sect. 4, the concept of the fuzzy efficient path is introduced and a FDEA based approach is proposed to solve the FMOSPP. The proposed approach is illustrated with two numerical examples in Sect. 5. In Sect. 6, the proposed approach is compared with the extended version of goal programming (GP) approach. Finally, conclusions are presented in Sect. 7.

\section{Mathematical background}

In the following subsections, preliminaries needed to introduce the proposed method to solve the FMOSPP are presented [37-41].

\section{Some basic definitions on fuzzy numbers}

Fuzzy sets theory was first introduced by Zadeh [42] to deal with the uncertainty of human judgment (Wang et al. [43]). Each fuzzy set is an extension of a crisp set that allows partial membership between 0 and 1. A fuzzy set on the real line that satisfies some conditions is called a fuzzy number. Here, some basic definitions and basic fuzzy arithmetic operations on the fuzzy numbers are reviewed [44].

Definition 2.1 A fuzzy set $A$ in $\mathbb{R}$ (real line) is defined to be a set of ordered pairs $A=\left\{\left(x, \mu_{A}(x)\right) \mid x \in \mathbb{R}\right\}$, where $\mu_{A}(x)$ is called the membership function for the fuzzy set.

Definition 2.2 A fuzzy set A is called normal if there is at least one point $x \in \mathbb{R}$ such that $\mu_{A}(x)=1$.

Definition 2.3 A fuzzy set A on $\mathbb{R}$ is called convex if for any $x, y \in \mathbb{R}$ and any $\lambda \in[0,1]$ the following inequality is satisfied:

$\mu_{A}(\lambda x+(1-\lambda) y) \leq \min \left\{\mu_{A}(x), \mu_{A}(y)\right\}$.

Definition 2.4 A fuzzy set $\tilde{A}$ on the real line that satisfies the conditions of normality and convexity is called a fuzzy number.

Definition 2.5 A fuzzy number $\tilde{A}$, denoted by $\tilde{A}=\left(a_{l}, a_{m}, a_{u}\right)$, is called a triangular fuzzy number if its membership function is given by relation (2):

$\mu_{\tilde{A}}(x)= \begin{cases}\frac{x-a_{l}}{a_{m}-a_{l}} & \text { for } a_{l} \leq x \leq a_{m} \\ \frac{a_{u}-x}{a_{u}-a_{m}} & \text { for } a_{m} \leq x \leq a_{u}\end{cases}$ 
Definition 2.6 A triangular fuzzy number $\tilde{A}=\left(a_{l}, a_{m}, a_{u}\right)$ is said to be a non-negative (positive) triangular fuzzy number if and only if $a_{l} \geq 0\left(a_{l}>0\right)$.

Definition 2.7 Two triangular fuzzy numbers $\tilde{A}=\left(a_{l}, a_{m}, a_{u}\right)$ and $\tilde{B}=\left(b_{l}, b_{m}, b_{u}\right)$ is said to be equal if, and only if, $a_{l}=b_{l}, a_{m}=b_{m}, a_{u}=b_{u}$.

Definition 2.8 Let $\tilde{A}=\left(a_{l}, a_{m}, a_{u}\right)$ and $\tilde{B}=\left(b_{l}, b_{m}, b_{u}\right)$ be two positive triangular fuzzy numbers and $t$ is a non-negative scaler. Then the basic fuzzy arithmetic operations along with the scaler multiplication are defined as follows:

$$
\begin{array}{cl}
\text { Addition: } & \tilde{A}+\tilde{B}=\left(a_{l}+b_{l}, a_{m}+b_{m}, a_{u}+b_{u}\right) \\
\text { Subtraction: } & \tilde{A}-\tilde{B}=\left(a_{l}-b_{u}, a_{m}-b_{m}, a_{u}-b_{l}\right) \\
\text { Multiplication: } & \tilde{A} \times \tilde{B}=\left(a_{l} \cdot b_{l}, a_{m}, b_{m}, a_{u} \cdot b_{u}\right) \\
\text { Division: } & \tilde{A} / \tilde{B}=\left(a_{l} / b_{u}, a_{m} / b_{m}, a_{u} / b_{l}\right), \\
\text { Scaler multiplication: } t \tilde{A}=\left(t a_{l}, t a_{m}, t a_{u}\right) .
\end{array}
$$

Definition 2.9 The $\alpha$-cutof fuzzy set $\tilde{A}$ on $\mathbb{R}$, denoted by $[\tilde{A}]_{\alpha}$ is a crisp set that contains all the elements of $\mathbb{R}$ whose membership degree in $\tilde{A}$ are greater than or equal to the value of $\alpha$, that is,

$[\tilde{A}]_{\alpha}=\left\{x \in \mathbb{R} \mid \mu_{A}(x) \geq \alpha\right\}$.

Theorem 2.10 The $\alpha$-cut of the triangular fuzzy number $\tilde{A}=$ $\left(a_{l}, a_{m}, a_{u}\right)$ is the closed interval as relation (5) [37]:

$$
[\tilde{A}]_{\alpha}=\left[(\tilde{A})_{\alpha}^{l},(\tilde{A})_{\alpha}^{u}\right]=\left[a_{l}+\left(a_{m}-a_{l}\right) \alpha, a_{u}-\left(a_{u}-a_{m}\right) \alpha\right] .
$$

\section{Mathematical model of FDEA}

The DEA is a useful non-parametric method that allows handling multiple outputs and inputs at the same time. Mathematically, DEA is a linear programming-based technique for evaluating the relative efficiency of a set of DMUs with multiple inputs and outputs. A major stated advantage of the DEA is that it does not require any prior assumptions on underlying functional relationships between inputs and outputs [45]. In classic DEA models, input and output data are assumed to be crisp values. However, in many real-world applications, the available data are imprecise or vague. This issue may result from unquantifiable, incomplete, missed information, etc. In recent years, much effort has been made on the theoretical development of Fuzzy DEA (FDEA) to deal with this impreciseness and ambiguity in DEA.

Suppose we wish to evaluate the efficiencies of $n$ homogeneous DMUs. Each $D M U_{j}(j=1, \ldots, n)$ produces $s$ different fuzzy outputs $\tilde{y}_{j}=\left(\tilde{y}_{1 j}, \ldots, \tilde{y}_{s j}\right)$, using $m$ different fuzzy inputs $\tilde{x}_{j}=\left(\tilde{x}_{1 j}, \ldots, \tilde{x}_{m j}\right)$. The multiplier form of the input-oriented CCR model [36] for evaluating the relative efficiency of $D M U_{p}$ is given by model (6):

$$
\begin{aligned}
\operatorname{Max} \tilde{\theta}_{p}= & \sum_{r=1}^{s} u_{r} \tilde{y}_{r p} \\
\text { s.t. } & \sum_{i=1}^{m} v_{i} \tilde{x}_{i p}=\tilde{1}, \\
& \sum_{r=1}^{s} u_{r} \tilde{y}_{r j}-\sum_{i=1}^{m} v_{i} \tilde{x}_{i j} \leq \tilde{0}, \quad j=1, \ldots, n, \\
& u_{r}, v_{i} \geq 0, \quad r=1, \ldots, s, i=1, \ldots, m,
\end{aligned}
$$

where ${ }^{\prime}$, indicates the fuzziness and $u_{r} \mathrm{~s}(r=1, \ldots, s)$ along with the $v_{i} \mathrm{~s}(i=1, \ldots, m)$ are the weights assigned to the outputs and inputs, respectively.

In this paper, to transform the Fuzzy Multi-Objective Shortest Path Problem (FMOSPP) into a single objective Fuzzy Shortest Path Problem (FSPP), some FDEA models will need to be solved. There exist several methods to solve the FDEA model (6) (e.g., [27,28,30,31]). Nonetheless, we use the proposed approach by Saati et al. [35] which is a fuzzy version of the CCR model [36] with the asymmetrical triangular fuzzy numbers. The basis of this approach is to transform each FDEA model into an interval model. In fact, using this approach, the FDEA model (6) is converted into a possibilistic programming problem and then transformed into a crisp linear programming model based on $\alpha$-cut. In this way, by solving the linear programming problem for a given $\alpha$-cut, it will be possible to generate a reliable and robust solution for the possibilistic mathematical programming problem.

\section{Fuzzy multi-objective shortest path problem (FMOSPP)}

The aim of the MOSPP is to find the shortest path between a pair of source and destination node in a network with respect to the multiple predefined objectives. In other words, there are two or more objective functions that must be optimized when selecting a path. When at least one of the parameters of a MOSPP is represented in terms of fuzzy numbers, it is called FMOSPP. The fuzzy numbers can appear in different parameters simultaneously and also be of different types such as normal, triangular or trapezoidal fuzzy numbers along with crisp numbers. Throughout the paper, without loss of generality, we consider all the parameters of the FMOSPP as non-negative triangular fuzzy numbers.

For more details, consider a directed graph $G=(V, A)$, where the vertices or nodes of the graph are contained in the 
Fig. 1 Arc $(i, j)$ including $p$ fuzzy attributes as a DMU with $k$ inputs and $s$ outputs $(k+s=p)$

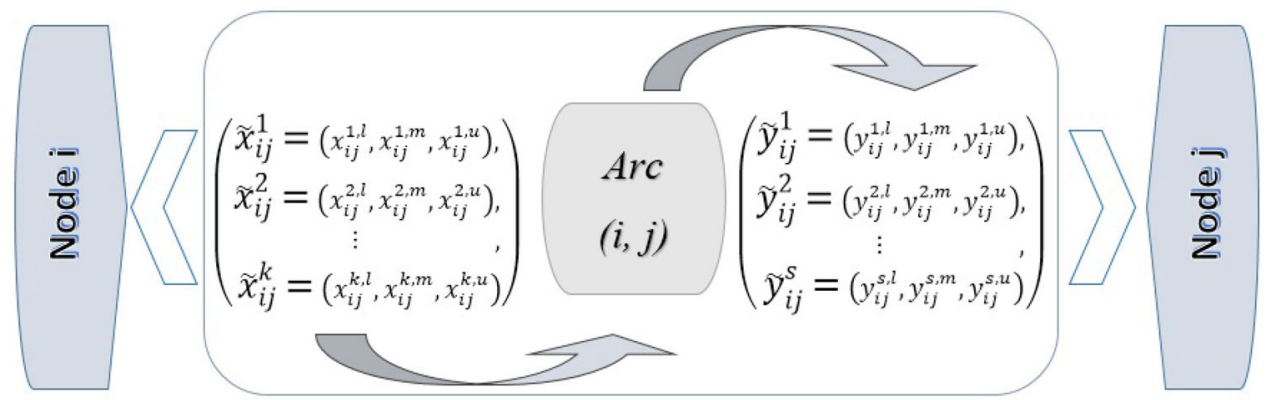

set $V=\{1,2, \ldots, n\}$ and the $m$ arcs or edges join the nodes are contained in the set $A=\{(i, j) \mid(i, j)$ is an arc in $G\}$. Corresponding to each arc $(i, j)$, there are $p$ associated non-negative fuzzy attributes $\tilde{c}_{i j}^{k}(k=1, \ldots, p)$. Thus, the FMOSPP has $p$ fuzzy objectives that need to be minimized and maximized. The problem is to find a best feasible path from the source node 1 to the destination node $n$ such that each node in this path must be considered just one time. Mathematical model of this FMOSPP can be written as model (7):

$$
\begin{aligned}
\text { Optimize } z= & \left(\sum_{(i, j) \in A} \tilde{c}_{i j}^{1} x_{i j}, \ldots, \sum_{(i, j) \in A} \tilde{c}_{i j}^{p} x_{i j}\right) \\
\text { s.t. } & \sum_{j:(1, j) \in A} x_{1 j}-\sum_{l:(l, 1) \in A} x_{l 1}=1 \\
& \sum_{j:(i, j) \in A} x_{i j}-\sum_{l:(l, i) \in A} x_{l i}=0, \quad \text { for all }(i, j) \in A-\{1, n\}, \\
& \sum_{j:(n, j) \in A} x_{n j}-\sum_{l:(l, n) \in A} x_{l n}=-1 \\
& x_{i j}=0,1, \quad \text { for all }(i, j) \in A
\end{aligned}
$$

where $x_{i j}=1$ or $x_{i j}=0$ indicates that the $\operatorname{arc}(i, j)$ is in the path or not, respectively. There are a few methods to solve the FMOSPP (7) (e.g. [13-15]). In Section 4, we propose a DEA-based approach to solve the FMOSPP (7).

\section{Fuzzy efficient path}

Consider the FMOSPP (7) with $p$ fuzzy objectives corresponding to the undirected network $G=(V, A)$ that needs to be minimized and maximized. To find an optimal solution to this problem, each arc of the network is considered as a DMU. The arc attributes corresponding to the fuzzy objectives that should be minimized are considered as fuzzy inputs and the attributes corresponding to the fuzzy objectives that should be maximized are considered as fuzzy outputs of the DMUs. The aim of the proposed approach is to convert the $p$ fuzzy attributes associated with each arc into a unique fuzzy attribute. In this way, the given FMOSPP (7) is transformed into a single objective fuzzy SPP. Then an efficient path with the maximum fuzzy efficiency is determined as an optimal solution to this problem. To this end, based on the FDEA, two fuzzy efficiency scores are obtained corresponding to each arc as a criteria of its relative performance. After that, the mean of the fuzzy efficiency scores is used to derive a new fuzzy attribute for each arc.

For a detailed description of the proposed method, let $\tilde{X}_{i j}=\left(\tilde{x}_{i j}^{1}, \ldots, \tilde{x}_{i j}^{k}\right)$ represents the fuzzy input vector of the $\operatorname{arc}(i, j) \in A$, where $\tilde{x}_{i j}^{t}=\left(x_{i j}^{t, l}, x_{i j}^{t, m}, x_{i j}^{t, u}\right)$ is a nonnegative triangular fuzzy number for all $t=1, \ldots, k$. In addition, suppose that $\tilde{Y}_{i j}=\left(\tilde{y}_{i j}^{1}, \ldots, \tilde{y}_{i j}^{s}\right)$ represents the fuzzy output vector of the $\operatorname{arc}(i, j) \in A$ where $\tilde{y}_{i j}^{r}=$ $\left(y_{i j}^{r, l}, y_{i j}^{r, m}, y_{i j}^{r, u}\right)$ is also a non-negative triangular fuzzy number for all $r=1, \ldots, s$. In other words, each $\operatorname{arc}(i, j) \in A$ has $k$ fuzzy inputs and $s$ fuzzy outputs such that $p=k+s$. Figure 1 shows the structure of each arc as a DMU.

Now, to solve the FMOSPP (7), the following steps should be taken:

Step 1. For each $\operatorname{arc}(i, j) \in A$, consider all the existing $\operatorname{arc}(i, h) \in A$. Then with the node $i$ as a target and changing the $h$, subject to $(i, h) \in A$, obtain the fuzzy relative efficiency of the $\operatorname{arc}(i, j) \in A$, i.e. $\tilde{E}_{i j}^{1}$, as the optimal objective value of the FDEA model (8):

$$
\begin{aligned}
\tilde{E}_{i j}^{1}=\max & \sum_{r=1}^{s} u_{r} \tilde{y}_{i j}^{r} \\
\text { s.t. } & \sum_{t=1}^{k} v_{t} \tilde{x}_{i j}^{t}=\tilde{1}, \\
& \sum_{r=1}^{s} u_{r} \tilde{y}_{i h}^{r}-\sum_{t=1}^{k} v_{t} \tilde{x}_{i h}^{t} \leq \tilde{0}, \forall h ;(i, h) \in A, \\
& u_{r} \geq 0, r=1, \ldots, s, v_{t} \geq 0, t=1, \ldots, k .
\end{aligned}
$$

By solving model (8) for all $i$ and $j$, obtain the fuzzy relative efficiency scores $\tilde{E}_{i j}^{1} \mathrm{~s}$ related to all of the existing arcs in $A$. How to solve model (8) will be discussed later. 
Step 2. For each arc $(i, j) \in A$, consider all the existing $\operatorname{arc}(h, j) \in A$. Then, with the node $j$ as a target and changing the $h$, subject to $(h, j) \in A$, obtain the fuzzy relative efficiency of the $\operatorname{arc}(i, j) \in A$, denoted by $\tilde{E}_{i j}^{2}$, as the optimal objective value of the FDEA model (9):

$$
\begin{aligned}
\tilde{E}_{i j}^{2}=\max & \sum_{r=1}^{s} u_{r} \tilde{y}_{i j}^{r} \\
\text { s.t. } & \sum_{t=1}^{k} v_{t} \tilde{x}_{i j}^{t}=\tilde{1}, \\
& \sum_{r=1}^{s} u_{r} \tilde{y}_{h j}^{r}-\sum_{t=1}^{k} v_{t} \tilde{x}_{h j}^{t} \leq \tilde{0}, \forall h ;(h, j) \in A, \\
& u_{r} \geq 0, r=1, \ldots, s, v_{t} \geq 0, t=1, \ldots, k .
\end{aligned}
$$

Step 3. By having the efficiency scores $\tilde{E}_{i j}^{1}$ and $\tilde{E}_{i j}^{2}$, obtain the unique fuzzy efficiency score $\tilde{E}_{i j}^{*}$ for the arc $(i, j)$ from the relation (10) as follows:

$$
\tilde{E}_{i j}^{*}=\frac{\tilde{E}_{i j}^{1}+\tilde{E}_{i j}^{2}}{2} .
$$

Step 4. Replace the existing multiple fuzzy attributes $\left(\tilde{c}_{i j}^{1}, \ldots, \tilde{c}_{i j}^{p}\right)$ with the single positive fuzzy attribute $\tilde{E}_{i j}^{*}$, for all $i$ and $j$. Then solve the single objective FSPP (11) as follows:

$$
\begin{aligned}
\operatorname{Max} z= & \sum_{(i, j) \in A} \tilde{E}_{i j}^{*} x_{i j} \\
\text { s.t. } & \sum_{j:(1, j) \in A} x_{1 j}-\sum_{l:(l, 1) \in A} x_{l 1}=1 \\
& \sum_{j:(i, j) \in A} x_{i j}-\sum_{l:(l, i) \in A} x_{l i}=0, \text { for all }(i, j) \in A-\{1, n\}, \\
& \sum_{j:(n, j) \in A} x_{n j}-\sum_{l:(l, n) \in A} x_{l n}=-1 \\
& x_{i j}=0,1, \quad \text { for all }(i, j) \in A .
\end{aligned}
$$

To solve the FDEA model (8) and (9), we follow the Saati et al.'s [35] approach. For this purpose, consider model (8) in its detailed form as model (12):

$$
\begin{aligned}
\tilde{E}_{i j}^{1}=\max & \sum_{r=1}^{s} u_{r}\left(y_{i j}^{r, l}, y_{i j}^{r, m}, y_{i j}^{r, u}\right) \\
\text { s.t. } & \sum_{t=1}^{k} v_{t}\left(x_{i j}^{t, l}, x_{i j}^{t, m}, x_{i j}^{t, u}\right)=\left(1^{l}, 1^{m}, 1^{u}\right), \\
& \sum_{r=1}^{s} u_{r}\left(y_{i h}^{r, l}, y_{i h}^{r, m}, y_{i h}^{r, u}\right)
\end{aligned}
$$

$$
\begin{aligned}
& -\sum_{t=1}^{k} v_{t}\left(x_{i h}^{t, l}, x_{i h}^{t, m}, x_{i h}^{t, u}\right) \leq\left(0^{l}, 0^{m}, 0^{u}\right) \\
& \quad \forall h ;(i, h) \in A, \\
& u_{r}, v_{t} \geq 0, \quad r=1, \ldots, s, t=1, \ldots, k
\end{aligned}
$$

where $1^{l}=1^{m}=1^{u}$ is considered equal to 1 and $0^{l}=0^{m}=$ $0^{u}=0$, to ensure that the efficiency values are not greater than 1. Model (12) is a possibilistic linear programming. By introducing $\alpha$-cuts of the objective function and constraints, this model can be written as model (13):

$$
\begin{gathered}
E_{i j}^{1, \alpha}=\max \sum_{r=1}^{s} u_{r}\left[y_{i j}^{r, l}+\left(y_{i j}^{r, m}-y_{i j}^{r, l}\right) \alpha, y_{i j}^{r, u}-\left(y_{i j}^{r, u}-y_{i j}^{r, m}\right) \alpha\right] \\
\text { s.t. } \sum_{t=1}^{k} v_{t}\left[x_{i j}^{t, l}+\left(x_{i j}^{t, m}-x_{i j}^{t, l}\right) \alpha, x_{i j}^{t, u}-\left(x_{i j}^{t, u}-x_{i j}^{t, m}\right) \alpha\right]=[1,1], \\
\sum_{r=1}^{s} u_{r}\left[y_{i h}^{r, l}+\left(y_{i h}^{r, m}-y_{i h}^{r, l}\right) \alpha, y_{i h}^{r, u}-\left(y_{i h}^{r, u}-y_{i h}^{r, m}\right) \alpha\right]- \\
\sum_{t=1}^{k} v_{t}\left[x_{i h}^{t, l}+\left(x_{i h}^{t, m}-x_{i h}^{t, l}\right) \alpha, x_{i h}^{t, u}-\left(x_{i h}^{t, u}\right.\right. \\
\left.\left.-x_{i h}^{t, m}\right) \alpha\right] \leq[0,0] \\
\forall h ;(i, h) \in A, \\
u_{r}, v_{t} \geq 0, \quad r=1, \ldots, s, t=1, \ldots, k .
\end{gathered}
$$

Since all of the coefficients and RHSs are stated as intervals, model (13) involves treating an interval problem and, hence, cannot be treated by standard methods without further transformations. There are several methods to solve this model that most of them compare the intervals in both sides of the constraints with each other. However, according to Saati et al. [35], instead of comparing the intervals, the variables in the intervals are defined such that they satisfy the set of constraints, and, at the same time the objective function is maximized. To this end, consider the variables $\hat{x}_{i h}^{t}$ and $\hat{y}_{i h}^{r}$ according to the relation:

$$
\begin{aligned}
& \hat{x}_{i h}^{t} \in\left[x_{i h}^{t, l}+\left(x_{i h}^{t, m}-x_{i h}^{t, l}\right) \alpha, x_{i h}^{t, u}-\left(x_{i h}^{t, u}-x_{i h}^{t, m}\right) \alpha\right], \\
& \forall h ;(i, h) \in A, t=1, \ldots, k, \\
& \hat{y}_{i h}^{r} \in\left[y_{i h}^{r, l}+\left(y_{i h}^{r, m}-y_{i h}^{r, l}\right) \alpha, y_{i h}^{r, u}-\left(y_{i h}^{r, u}-y_{i h}^{r, m}\right) \alpha\right], \\
& \quad \forall h ;(i, h) \in A, r=1, \ldots, s .
\end{aligned}
$$

By substituting the variables $\hat{x}_{i h}^{t}$ and $\hat{y}_{i h}^{r}$, model (13) is transformed to model (15) as follows:

$$
\begin{aligned}
E_{i j}^{1, \alpha}=\max & \sum_{r=1}^{s} u_{r} \hat{y}_{i j}^{r} \\
\text { s.t. } & \sum_{t=1}^{k} v_{t} \hat{x}_{i j}^{t}=1, \\
& \sum_{r=1}^{s} u_{r} \hat{y}_{i h}^{r}-\sum_{t=1}^{k} v_{t} \hat{x}_{i h}^{t} \leq 0, \forall h ;(i, h) \in A,
\end{aligned}
$$


$y_{i j}^{r, l}+\left(y_{i j}^{r, m}-y_{i j}^{r, l}\right) \alpha \leq \hat{y}_{i j}^{r} \leq y_{i j}^{r, u}-\left(y_{i j}^{r, u}-y_{i j}^{r, m}\right) \alpha, \forall h ;(i, h) \in A, r=1, \ldots, s$,

$x_{i j}^{t, l}+\left(x_{i j}^{t, m}-x_{i j}^{t, l}\right) \alpha \leq \hat{x}_{i j}^{t} \leq x_{i j}^{t, u}-\left(x_{i j}^{t, u}-x_{i j}^{t, m}\right) \alpha, \forall h ;(i, h) \in A, t=1, \ldots, k$,

$$
u_{r}, v_{t} \geq 0, \quad r=1, \ldots, s, t=1, \ldots, k \text {. }
$$

Model (15) is a non-linear programming problem. To transform this model to a linear programming model, the variable substitutions can be used as the relation:

$\bar{x}_{i h}^{t}=v_{t} \hat{x}_{i h}^{t}, \bar{y}_{i h}^{r}=u_{r} \hat{y}_{i h}^{r}$

$\forall h ;(i, h) \in A, t=1, \ldots, k, r=1, \ldots, s$.

According to relation (16), an optimization point of model (13) can be obtained from the parametric linear programming problem (17), while $\alpha \in(0,1]$ is its parameter:

$$
\begin{aligned}
& E_{i j}^{1, \alpha}=\max \sum_{r=1}^{s} \bar{y}_{i j}^{r} \\
& \text { s.t. } \sum_{t=1}^{k} \bar{x}_{i j}^{t}=1 \\
& \sum_{r=1}^{s} \bar{y}_{i h}^{r}-\sum_{t=1}^{k} \bar{x}_{i h}^{t} \leq 0, \forall h ;(i, h) \in A \\
& v_{t}\left(x_{i h}^{t, l}+\left(x_{i h}^{t, m}-x_{i h}^{t, l}\right) \alpha\right) \leq \bar{x}_{i h}^{t} \leq v_{t}\left(x_{i h}^{t, u}-\left(x_{i h}^{t, u}-x_{i h}^{t, m}\right) \alpha\right) \\
& \quad \forall h ;(i, h) \in A t=1, \ldots, k \\
& u_{r}\left(y_{i h}^{r, l}+\left(y_{i h}^{r, m}-y_{i h}^{r, l}\right) \alpha\right) \leq \bar{y}_{i h}^{r} \leq u_{r}\left(y_{i h}^{r, u}-\left(y_{i h}^{r, u}-y_{i h}^{r, m}\right) \alpha\right) \\
& \forall h ;(i, h) \in A r=1, \ldots, s, \\
& u_{r}, v_{t} \geq 0, \quad r=1, \ldots, s, t=1, \ldots, k
\end{aligned}
$$

Similarly, model (9) can be solved following the Saati et al.'s [35] approach. Therefore, by solving model (17) for all $(i, j) \in A$, the fuzzy efficiency score $E_{i j}^{*, \alpha}$ can be obtained from the relation (18), corresponding to each particular $\alpha \in$ $(0,1]$ :

$E_{i j}^{*, \alpha}=\frac{E_{i j}^{1, \alpha}+E_{i j}^{2, \alpha}}{2}$.

Finally, an efficient path corresponding to the desired ' $\alpha$ ' can be determined by solving model (19):

$$
\begin{aligned}
& \operatorname{Max} z^{\alpha}= \sum_{(i, j) \in A} E_{i j}^{*, \alpha} x_{i j} \\
& \text { s.t. } \sum_{j:(1, j) \in A} x_{1 j}-\sum_{l:(l, 1) \in A} x_{l 1}=1 \\
& \sum_{j:(i, j) \in A} x_{i j}-\sum_{l:(l, i) \in A} x_{l i}=0, \\
& \text { for all }(i, j) \in A-\{1, n\}, \\
& \sum_{j:(n, j) \in A} x_{n j}-\sum_{l:(l, n) \in A} x_{l n}=-1 \\
& x_{i j}=0,1, \quad \text { for all }(i, j) \in A .
\end{aligned}
$$

Theorem 4.1 For any $\alpha_{1}, \alpha_{2} \in(0,1]$ such that $\alpha_{1} \leq \alpha_{2}$, we have $E_{i j}^{1, \alpha_{2}} \leq E_{i j}^{1, \alpha_{1}}$; where, $E_{i j}^{1, \alpha_{1}}$ and $E_{i j}^{1, \alpha_{2}}$ are the optimum objective function values of model (17) at $\alpha=\alpha_{1}$ and $\alpha=\alpha_{2}$, respectively.

Proof Suppose that $u_{r}^{*}, v_{t}^{*}, \bar{y}_{i h}^{r *}$ and $\bar{x}_{i h}^{t *}(\forall h ;(i, h) \in A, r=$ $1, \ldots, s, t=1, \ldots, k)$ be an optimal solution of model (17) at $\alpha=\alpha_{2}$. Therefore, relations (20) to (22) hold:

$$
\begin{aligned}
& \left.\begin{array}{l}
\sum_{t=1}^{k} \bar{x}_{i j}^{* *}=1, \\
\sum_{r=1}^{s} \bar{y}_{i h}^{* *}-\sum_{t=1}^{k} \bar{x}_{i h}^{t *} \leq 0, \forall h ;(i, h) \in A,
\end{array}\right\} \\
& v_{t}^{*}\left(x_{i h}^{t, l}+\left(x_{i h}^{t, m}-x_{i h}^{t, l}\right) \alpha_{2}\right) \leq \bar{x}_{i h}^{t *} \leq v_{t}^{*}\left(x_{i h}^{t, u}-\left(x_{i h}^{t, u}-x_{i h}^{t, m}\right) \alpha_{2}\right) \\
& \forall h ;(i, h) \in A t=1, \ldots, k \text {, } \\
& \begin{array}{c}
u_{r}^{*}\left(y_{i h}^{r, l}+\left(y_{i h}^{r, m}-y_{i h}^{r, l}\right) \alpha_{2}\right) \leq \bar{y}_{i h}^{r *} \leq u_{r}^{*}\left(y_{i h}^{r, u}-\left(y_{i h}^{r, u}-y_{i h}^{r, m}\right) \alpha_{2}\right), \\
\forall h ;(i, h) \in A r=1, \ldots, s,
\end{array} \\
& u_{r}^{*}, v_{t}^{*} \geq 0, \quad r=1, \ldots, s, t=1, \ldots, k .
\end{aligned}
$$

On the other hand, for $\alpha_{1} \leq \alpha_{2}$, relations (23) and (24) clearly hold:

$$
\begin{gathered}
\left(x_{i h}^{t, l}+\left(x_{i h}^{t, m}-x_{i h}^{t, l}\right) \alpha_{1}\right) \leq\left(x_{i h}^{t, l}+\left(x_{i h}^{t, m}-x_{i h}^{t, l}\right) \alpha_{2}\right) \leq \\
\left(x_{i h}^{t, u}-\left(x_{i h}^{t, u}-x_{i h}^{t, m}\right) \alpha_{2}\right) \leq\left(x_{i h}^{t, u}-\left(x_{i h}^{t, u}-x_{i h}^{t, m}\right) \alpha_{1}\right), \\
\forall h ;(i, h) \in A t=1, \ldots, k, \\
\left(y_{i h}^{r, l}+\left(y_{i h}^{r, m}-y_{i h}^{r, l}\right) \alpha_{1}\right) \leq\left(y_{i h}^{r, l}+\left(y_{i h}^{r, m}-y_{i h}^{r, l}\right) \alpha_{2}\right) \leq \\
\quad\left(y_{i h}^{r, u}-\left(y_{i h}^{r, u}-y_{i h}^{r, m}\right) \alpha_{2}\right) \leq\left(y_{i h}^{r, u}-\left(y_{i h}^{r, u}-y_{i h}^{r, m}\right) \alpha_{1}\right), \\
\forall h ;(i, h) \in A r=1, \ldots, s .
\end{gathered}
$$

Thus, relations (23) and (24) lead to the relation (25):

$$
\left.\begin{array}{r}
v_{t}^{*}\left(x_{i h}^{t, l}+\left(x_{i h}^{t, m}-x_{i h}^{t, l}\right) \alpha_{1}\right) \leq \bar{x}_{i h}^{t *} \leq v_{t}^{*}\left(x_{i h}^{t, u}-\left(x_{i h}^{t, u}-x_{i h}^{t, m}\right) \alpha_{1}\right) \\
\forall h ;(i, h) \in A t=1, \ldots, k, \\
u_{r}^{*}\left(y_{i h}^{r, l}+\left(y_{i h}^{r, m}-y_{i h}^{r, l}\right) \alpha_{1}\right) \leq \bar{y}_{i h}^{r *} \leq u_{r}^{*}\left(y_{i h}^{r, u}-\left(y_{i h}^{r, u}-y_{i h}^{r, m}\right) \alpha_{1}\right), \\
\forall h ;(i, h) \in A r=1, \ldots, s, .
\end{array}\right\}
$$

Therefore, from relations (20), (22) and (25), it is concluded that $u_{r}^{*}, v_{t}^{*}, \bar{y}_{i h}^{r *}$ and $\bar{x}_{i h}^{t *}(\forall h ;(i, h) \in A, r=1, \ldots, s, t=$ $1, \ldots, k)$ is a feasible solution for model (17) at $\alpha=\alpha_{1}$; then, it is clear that $E_{i j}^{1, \alpha_{2}} \leq E_{i j}^{1, \alpha_{1}}$.

Note that Theorem 4.1 is also true for $E_{i j}^{2, \alpha_{1}}$ and $E_{i j}^{2, \alpha_{2}}$. Thus, from Theorem 4.1 and relation (18), it is immediately concluded that for any $\alpha_{1}, \alpha_{2} \in(0,1]$ such that $\alpha_{1} \leq \alpha_{2}$, the inequality $E_{i j}^{*, \alpha_{2}} \leq E_{i j}^{*, \alpha_{1}}$ holds.

Note 4.2 The objection function $\sum_{(i, j) \in A} E_{i j}^{*, \alpha} x_{i j}$ in model (19) can be replaced with the $\sum_{(i, j) \in A}\left(1-E_{i j}^{*, \alpha}\right) x_{i j}$. In this way, model (19) is transformed into a classic single objective SPP with a minimization objective function that can be solved using any standard algorithm for solving the SPP such as Dijkstra's algorithm [48].

Note 4.3 Using the mentioned $\alpha$-cut approach, instead of a unique fuzzy efficient path, the efficient path table corresponding to different $\alpha \in(0,1]$ will be provided for the decision-maker. 


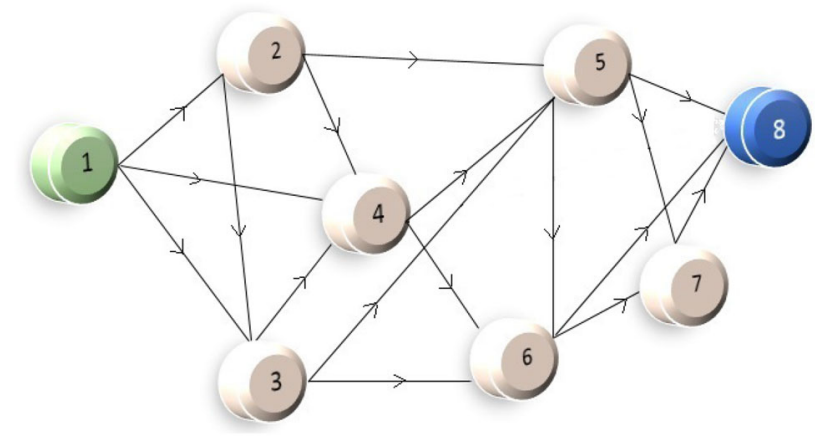

Fig. 2 A directed graph with 8 nodes and 18 arcs

\section{Numerical examples}

In this section, the proposed fuzzy DEA approach for solving fuzzy multi-objective shortest path problem is illustrated using two numerical examples.

Example 5.1 Consider the air transportation network $G_{1}=$ $\left(V_{1}, A_{1}\right)$ shown in Fig. 2 including 8 nodes (Airports) and 18 arcs (i.e, $\left|V_{1}\right|=8,\left|A_{1}\right|=18$ ).

Given the schedule of all airline flights and their flyingcosts, flying-delays and flying-safety, what is the efficient path to go from Airport 1 to Airport 8? The values of cost $\tilde{c}_{i j}^{1}$, time $\tilde{c}_{i j}^{2}$ and safety $\tilde{c}_{i j}^{3}$ based on triangular fuzzy numbers, denoted by $\left(a_{l}, a_{m}, a_{u}\right)$, are given in Table 1 .

Table 1 The input/output data of the graph $G_{1}=\left(V_{1}, A_{1}\right)$

\begin{tabular}{llll}
\hline Arcs & Cost & Time & Safety \\
\hline $1 \longrightarrow 2$ & $(65,70,80)$ & $(270,285,310)$ & $(0.25,0.28,0.35)$ \\
$1 \longrightarrow 3$ & $(42,98,153)$ & $(320,340,350)$ & $(0.06,0.15,0.28)$ \\
$1 \longrightarrow 4$ & $(51,60,75)$ & $(230,260,279)$ & $(0.3,0.35,0.38)$ \\
$2 \longrightarrow 3$ & $(85,90,98)$ & $(190,200,230)$ & $(0.58,0.6,0.62)$ \\
$2 \longrightarrow 4$ & $(62,65,70)$ & $(125,130,145)$ & $(0.3,0.38,0.4)$ \\
$2 \longrightarrow 5$ & $(38,40,49)$ & $(149,15,156)$ & $(0.42,0.45,0.46)$ \\
$3 \longrightarrow 4$ & $(98,110,120)$ & $(310,320,322)$ & $(0.85,0.9,0.92)$ \\
$3 \longrightarrow 5$ & $(75,80,86)$ & $(270,285,300)$ & $(0.25,0.3,0.31)$ \\
$3 \longrightarrow 6$ & $(89,90,91)$ & $(195,210,215)$ & $(0.75,0.82,0.85)$ \\
$4 \longrightarrow 5$ & $(30,35,39)$ & $(118,120,130)$ & $(0.55,0.56,0.65)$ \\
$4 \longrightarrow 6$ & $(82,85,96)$ & $(145,150,156)$ & $(0.22,0.26,0.29)$ \\
$5 \longrightarrow 6$ & $(25,26,30)$ & $(149,150,151)$ & $(0.74,0.85,0.91)$ \\
$5 \longrightarrow 7$ & $(70,90,92)$ & $(90,100,115)$ & $(0.88,0.9,0.95)$ \\
$5 \longrightarrow 8$ & $(145,150,155)$ & $(225,230,236)$ & $(0.64,0.68,0.7)$ \\
$6 \longrightarrow 7$ & $(218,220,230)$ & $(426,450,478)$ & $(0.9,0.95,0.96)$ \\
$6 \longrightarrow 8$ & $(79,80,84)$ & $(382,390,401)$ & $(0.83,0.85,0.89)$ \\
$7 \longrightarrow 8$ & $(120,125,128)$ & $(263,300,368)$ & $(0.8,0.87,0.9)$ \\
\hline
\end{tabular}

In this case, the following fuzzy three-objective shortest path problem is formulated to achieve the fuzzy efficient path:

$$
\begin{aligned}
\min & \sum_{(i, j) \in A_{1}} \tilde{c}_{i j}^{1} x_{i j} \\
\min & \sum_{(i, j) \in A_{1}} \tilde{c}_{i j}^{2} x_{i j} \\
\max & \sum_{(i, j) \in A_{1}} \tilde{c}_{i j}^{3} x_{i j} \\
\text { s.t. } & \sum_{j:} x_{1, j}-\sum_{l:(l, 1) \in A_{1}} x_{l 1}=1 \\
& \sum_{j:(i, j) \in A_{1}} x_{i j}-\sum_{l:(l, i) \in A_{1}} x_{l i}=0, \quad i=2,3,4,5,6,7, \\
& \sum_{j:} x_{8 j}-\sum_{l:(l, j) \in A_{1}} x_{l 8}=-1 \\
x_{i j}=0,1, \quad \text { for all }(i, j) \in A_{1} &
\end{aligned}
$$

For solving model (26), the fuzzy DEA approach proposed in this study is used. Thus, according to this approach, flyingcost and flying-delays are considered as inputs and flyingsafety is considered as output. Based on this approach, to achieve a fuzzy efficient path, the values of the efficiency scores $E_{i j}^{1, \alpha}$ and $E_{i j}^{2, \alpha}$ must first be obtained. To this end, the corresponding model (8) and (9) should be solved for the expected values of the $\alpha \in(0,1]$ determined by the decision maker. For example, $E_{13}^{1, \alpha}$ can be obtained as the optimum objection value of model (27) for a given $\alpha \in(0,1]$ :

$$
\begin{aligned}
\operatorname{Max} E_{13}^{\alpha, 1}= & \bar{y}_{13}^{1} \\
\text { s.t. } & \bar{x}_{13}^{1}+\bar{x}_{13}^{2}=1, \\
& \bar{y}_{1 h}^{1}-\bar{x}_{1 h}^{1}-\bar{x}_{1 h}^{2} \leq 0, h=2,3,4, \\
& v_{t}\left(x_{1 h}^{t, l}+\left(x_{1 h}^{t, m}-x_{1 h}^{t, l}\right) \alpha\right) \\
& \leq \bar{x}_{1 h}^{t} \leq v_{t}\left(x_{1 h}^{t, u}-\left(x_{1 h}^{t, u}-x_{1 h}^{t, m}\right) \alpha\right), \\
& h=2,3,4 t=1,2, \\
& u_{1}\left(y_{1 h}^{1, l}+\left(y_{1 h}^{1, m}-y_{1 h}^{1, l}\right) \alpha\right) \\
& \leq \bar{y}_{1 h}^{1} \leq u_{1}\left(y_{1 h}^{1, u}-\left(y_{1 h}^{1, u}-y_{1 h}^{1, m}\right) \alpha\right) \\
& \\
& u_{1}, v_{1}, v_{2} \geq 0,
\end{aligned}
$$

which is equivalent to model (28) according to the data of Table 1:

$$
\begin{aligned}
\operatorname{Max} & E_{13}^{\alpha, 1}=\bar{y}_{13}^{1} \\
\text { s.t. } & \bar{x}_{13}^{1}+\bar{x}_{13}^{2}=1, \\
& \bar{y}_{12}^{1}-\bar{x}_{12}^{1}-\bar{x}_{12}^{2} \leq 0, \\
& \bar{y}_{13}^{1}-\bar{x}_{13}^{1}-\bar{x}_{13}^{2} \leq 0, \\
& \bar{y}_{14}^{1}-\bar{x}_{14}^{1}-\bar{x}_{14}^{2} \leq 0,
\end{aligned}
$$




$$
\begin{aligned}
& v_{1}(65+(70-65) \alpha) \leq \bar{x}_{12}^{1} \leq v_{1}(80-(80-70) \alpha), \\
& v_{2}(270+(285-270) \alpha) \leq \bar{x}_{12}^{2} \leq v_{t}(310-(310-285) \alpha), \\
& v_{1}(42+(98-42) \alpha) \leq \bar{x}_{13}^{1} \leq v_{1}(153-(153-98) \alpha), \\
& v_{2}(320+(340-320) \alpha) \leq \bar{x}_{13}^{2} \leq v_{t}(350-(350-340) \alpha), \\
& v_{1}(51+(60-51) \alpha) \leq \bar{x}_{14}^{1} \leq v_{1}(75-(75-60) \alpha), \\
& v_{2}(230+(260-230) \alpha) \leq \bar{x}_{14}^{2} \leq v_{t}(279-(279-260) \alpha), \\
& u_{1}(0.25+(0.28-0.25) \alpha) \leq \bar{y}_{12}^{1} \leq u_{1}(0.35-(0.35-0.28) \alpha) \\
& u_{1}(0.06+(0.15-0.06) \alpha) \leq \bar{y}_{13}^{1} \leq u_{1}(0.28-(0.28-0.15) \alpha) \\
& u_{1}(0.3+(0.35-0.3) \alpha) \leq \bar{y}_{14}^{1} \leq u_{1}(0.38-(0.38-0.35) \alpha) \\
& u_{1}, v_{1}, v_{2} \geq 0 .
\end{aligned}
$$

Similarly, for a given $\alpha \in(0,1]$, the value of $E_{13}^{2, \alpha}$ can be obtained as the optimum objection value of model (29):

$$
\begin{aligned}
& \operatorname{Max} E_{13}^{\alpha, 2}= \bar{y}_{13}^{1} \\
& \text { s.t. } \bar{x}_{13}^{1}+\bar{x}_{13}^{2}=1, \\
& \bar{y}_{h 3}^{1}-\bar{x}_{h 3}^{1}-\bar{x}_{h 3}^{2} \leq 0, h=1,2, \\
& v_{t}\left(x_{h 3}^{t, l}+\left(x_{h 3}^{t, m}-x_{h 3}^{t, l}\right) \alpha\right) \\
& \leq \bar{x}_{h 3}^{t} \leq v_{t}\left(x_{h 3}^{t, u}-\left(x_{h 3}^{t, u}-x_{h 3}^{t, m}\right) \alpha\right), \\
& h=1,2, t=1,2, \\
& u_{1}\left(y_{h 3}^{1, l}+\left(y_{h 3}^{1, m}-y_{h 3}^{1, l}\right) \alpha\right) \\
& \leq \bar{y}_{h 3}^{1} \leq u_{1}\left(y_{h 3}^{1, u}-\left(y_{h 3}^{1, u}-y_{h 3}^{1, m}\right) \alpha\right), \\
& h=1,2,
\end{aligned}
$$$$
u_{1}, v_{1}, v_{2} \geq 0 \text {. }
$$

By obtaining the values of the $E_{i j}^{1, \alpha} \mathrm{s}$ and $E_{i j}^{2, \alpha}$ s for all $i$ and $j$, the values of the $E_{i j}^{*, \alpha} \mathrm{s}$ can be computed according to Relation (18). Tables 2, 3 and 4 represent the obtained results for the $E_{i j}^{1, \alpha} \mathrm{s}, E_{i j}^{2, \alpha} \mathrm{s}$ and $E_{i j}^{*, \alpha} \mathrm{s}$ corresponding to each $\alpha \in$ $\{0.1,0.2,0.3, \ldots, 0.9,1\}$, respectively.

Finally, by having the efficiency scores $E_{i j}^{*, \alpha} \mathrm{s}$, an efficient path of the graph $G_{1}=\left(V_{1}, A_{1}\right)$ can be obtained using the corresponding single objective SPP (19). The efficient path corresponding to $\alpha=0.1$ is $1 \rightarrow 4 \rightarrow 5 \rightarrow 6 \rightarrow 8$ and the efficient path corresponding to other ' $\alpha$ 's is $1 \rightarrow 2 \rightarrow$ $4 \rightarrow 5 \rightarrow 6 \rightarrow 8$. This shows that different efficient paths may result in different $\alpha \in(0,1]$. The membership function of the objective functions corresponding to the $\alpha=0.1$ and $\alpha=0.2=0.3=\cdots=0.9=1$ are shown in Figs. 3 and 4 , respectively.

Example 5.2 Consider the transportation network $G_{2}=$ $\left(V_{2}, A_{2}\right)$ shown in Fig. 5 including 14 nodes and 35 arcs $\left(\left|V_{2}\right|=14,\left|A_{2}\right|=35\right)$.

Table 5 shows the fuzzy values of Cost (Input 1), Time (Input 2) and Profit (Output 1) based on the triangular fuzzy numbers, denoted by $\left(a_{l}, a_{m}, a_{u}\right)$. The purpose is to find an efficient path from node 1 to node 14 .
According to Table 5, the computed value of $E_{i j}^{*, \alpha}$ s corresponding to each $\alpha \in\{0.1,0.2,0.3, \ldots, 0.9,1\}$ is listed in Table 6.

Now, by having the efficiency scores $E_{i j}^{*, \alpha} \mathrm{s}$, an efficient path of the graph $G_{2}=\left(V_{2}, A_{2}\right)$ can be obtained using the corresponding single objective SPP (19). The efficient path corresponding to each $\alpha=0.1=0.2=0.3=\ldots=0.9=$ 1 is $1 \rightarrow 3 \rightarrow 7 \rightarrow 9 \rightarrow 13 \rightarrow 14$. The membership functions of the objective functions (Cost, Time and Profit) corresponding to the obtained efficient path are given as [(880, 1090, 1210), $(145,185,215),(2160,2335,2500)]$.

\section{Comparison of results with fuzzy goal programming}

In this section, the performance of the proposed fuzzy DEA approach for solving FMOSPP (7) is compared with the extended version of goal programming (GP) approach.

GP is a popular approach for solving the multi-objective optimization problem by reducing it to a single objective one. The idea of GP is to minimize the distance between objective functions and an aspiration level vector either determined by the decision-maker or equals $\tilde{f}=\left(\tilde{f}_{1}^{*}, \tilde{f}_{2}^{*}, \ldots, \tilde{f}_{h}^{*}\right)$, where

$$
\begin{aligned}
\tilde{f}_{k}^{*}=\text { Optimize } & \sum_{(i, j) \in A} \tilde{c}_{i j}^{k} x_{i j} \\
\text { s.t. } & \sum_{j:(1, j) \in A} x_{i j}-\sum_{l:(l, 1) \in A} x_{l 1}=1, \\
& \sum_{j:(i, j) \in A} x_{i j}-\sum_{l:(l, i) \in A} x_{l i}=1, \\
& \text { for all }(i, j) \in A, i \neq 1, n, \\
& \sum_{j:(n, j) \in A} x_{i j}-\sum_{l:(l, n) \in A} x_{l 1}=-1, \\
& x_{i j}=0,1, \quad \text { for all }(i, j) \in A .
\end{aligned}
$$

Assume that $\tilde{n}_{t}=\left(n_{t}^{l}, n_{t}^{m}, n_{t}^{u}\right)$ and $\tilde{p}_{t}=\left(p_{t}^{l}, p_{t}^{m}, p_{t}^{u}\right)$ are the under deviations (achievement) and over deviations of the objectives $\tilde{f}_{t}=\sum_{(i, j) \in A} \tilde{c}_{i j}^{t} x_{i j}$ from their aspiration values $\tilde{f}_{t}^{*}$, respectively. Let $\tilde{f}_{t}=\sum_{(i, j) \in A} \tilde{c}_{i j}^{t} x_{i j}(t=1, \ldots, k)$ and $\tilde{f}_{t}=\sum_{(i, j) \in A} \tilde{c}_{i j}^{t} x_{i j}(t=k+1, \ldots, k+s)$ be those objective functions of FMOSPP (7) that should be minimized and maximized, respectively. Accordingly, the FMOSPP (7) is converted by GP into a minimization problem of the deviational variables which minimizes the sum of deviational variables:

$\min \sum_{t=1}^{k} \tilde{p}_{t}+\sum_{t=k+1}^{k+s} \tilde{n}_{t}$

s.t. 
Table 2 The values of the $E_{i j}^{1, \alpha} \mathrm{s}$ for different levelspc of $\alpha \in(0,1]$

\begin{tabular}{|c|c|c|c|c|c|c|c|c|c|c|}
\hline \multirow[t]{2}{*}{ Arcs } & \\
\hline & $\alpha=0.1$ & $\alpha=0.2$ & $\alpha=0.3$ & $\alpha=0.4$ & $\alpha=0.5$ & $\alpha=0.6$ & $\alpha=0.7$ & $\alpha=0.8$ & $\alpha=0.9$ & $\alpha=1$ \\
\hline $1 \longrightarrow 2$ & 1 & 1 & 1 & 1 & 0.97 & 0.91 & 0.85 & 0.81 & 0.77 & 0.73 \\
\hline $1 \longrightarrow 3$ & 1 & 1 & 0.91 & 0.76 & 0.63 & 0.53 & 0.45 & 0.41 & 0.37 & 0.33 \\
\hline $1 \longrightarrow 4$ & 1 & 1 & 1 & 1 & 1 & 1 & 1 & 1 & 1 & 1 \\
\hline $2 \longrightarrow 3$ & 1 & 1 & 1 & 1 & 1 & 1 & 1 & 1 & 1 & 1 \\
\hline $2 \longrightarrow 4$ & 1 & 1 & 1 & 1 & 1 & 1 & 1 & 1 & 0.99 & 0.97 \\
\hline $2 \longrightarrow 5$ & 1 & 1 & 1 & 1 & 1 & 1 & 1 & 1 & 1 & 1 \\
\hline $3 \longrightarrow 4$ & 1 & 1 & 1 & 1 & 1 & 0.98 & 0.96 & 0.93 & 0.91 & 0.89 \\
\hline $3 \longrightarrow 5$ & 0.49 & 0.48 & 0.47 & 0.46 & 0.45 & 0.44 & 0.43 & 0.42 & 0.42 & 0.41 \\
\hline $3 \longrightarrow 6$ & 1 & 1 & 1 & 1 & 1 & 1 & 1 & 1 & 1 & 1 \\
\hline $4 \longrightarrow 5$ & 1 & 1 & 1 & 1 & 1 & 1 & 1 & 1 & 1 & 1 \\
\hline $4 \longrightarrow 6$ & 0.46 & 0.45 & 0.44 & 0.43 & 0.42 & 0.41 & 0.4 & 0.39 & 0.38 & 0.37 \\
\hline $5 \longrightarrow 6$ & 1 & 1 & 1 & 1 & 1 & 1 & 1 & 1 & 1 & 1 \\
\hline $5 \longrightarrow 7$ & 1 & 1 & 1 & 1 & 1 & 1 & 1 & 1 & 1 & 1 \\
\hline $5 \longrightarrow 8$ & 0.44 & 0.43 & 0.43 & 0.42 & 0.41 & 0.4 & 0.4 & 0.39 & 0.38 & 0.38 \\
\hline $6 \longrightarrow 7$ & 1 & 1 & 1 & 1 & 1 & 1 & 0.99 & 0.98 & 0.97 & 0.96 \\
\hline $6 \longrightarrow 8$ & 1 & 1 & 1 & 1 & 1 & 1 & 1 & 1 & 1 & 1 \\
\hline $7 \longrightarrow 8$ & 1 & 1 & 1 & 1 & 1 & 1 & 1 & 1 & 1 & 1 \\
\hline
\end{tabular}

Table 3 The values of the $E_{i j}^{2, \alpha} \mathrm{s}$ for different levels of $\alpha \in(0,1]$

\begin{tabular}{|c|c|c|c|c|c|c|c|c|c|c|}
\hline \multirow[t]{2}{*}{ Arcs } & \\
\hline & $\alpha=0.1$ & $\alpha=0.2$ & $\alpha=0.3$ & $\alpha=0.4$ & $\alpha=0.5$ & $\alpha=0.6$ & $\alpha=0.7$ & $\alpha=0.8$ & $\alpha=0.9$ & $\overline{\alpha=1}$ \\
\hline $1 \longrightarrow 2$ & 1 & 1 & 1 & 1 & 1 & 1 & 1 & 1 & 1 & 1 \\
\hline $1 \longrightarrow 3$ & 0.92 & 0.77 & 0.66 & 0.56 & 0.48 & 0.41 & 0.36 & 0.31 & 0.26 & 0.23 \\
\hline $1 \longrightarrow 4$ & 1 & 0.96 & 0.93 & 0.89 & 0.86 & 0.82 & 0.79 & 0.76 & 0.73 & 0.71 \\
\hline $2 \longrightarrow 3$ & 1 & 1 & 1 & 1 & 1 & 1 & 1 & 1 & 1 & 1 \\
\hline $2 \longrightarrow 4$ & 1 & 1 & 1 & 1 & 1 & 1 & 1 & 1 & 1 & 1 \\
\hline $2 \longrightarrow 5$ & 0.84 & 0.82 & 0.81 & 0.79 & 0.78 & 0.76 & 0.75 & 0.73 & 0.72 & 0.7 \\
\hline $3 \longrightarrow 4$ & 1 & 1 & 1 & 1 & 1 & 1 & 1 & 1 & 1 & 1 \\
\hline $3 \longrightarrow 5$ & 0.29 & 0.28 & 0.27 & 0.27 & 0.26 & 0.26 & 0.25 & 0.24 & 0.24 & 0.23 \\
\hline $3 \longrightarrow 6$ & 0.86 & 0.84 & 0.82 & 0.8 & 0.78 & 0.76 & 0.74 & 0.72 & 0.7 & 0.69 \\
\hline $4 \longrightarrow 5$ & 1 & 1 & 1 & 1 & 1 & 1 & 1 & 1 & 1 & 1 \\
\hline $4 \longrightarrow 6$ & 0.39 & 0.38 & 0.37 & 0.36 & 0.35 & 0.34 & 0.33 & 0.32 & 0.31 & 0.3 \\
\hline $5 \longrightarrow 6$ & 1 & 1 & 1 & 1 & 1 & 1 & 1 & 1 & 1 & 1 \\
\hline $5 \longrightarrow 7$ & 1 & 1 & 1 & 1 & 1 & 1 & 1 & 1 & 1 & 1 \\
\hline $5 \longrightarrow 8$ & 1 & 1 & 1 & 1 & 1 & 1 & 1 & 1 & 1 & 1 \\
\hline $6 \longrightarrow 7$ & 0.45 & 0.45 & 0.44 & 0.44 & 0.44 & 0.43 & 0.43 & 0.43 & 0.43 & 0.42 \\
\hline $6 \longrightarrow 8$ & 1 & 1 & 1 & 1 & 1 & 1 & 1 & 1 & 1 & 1 \\
\hline $7 \longrightarrow 8$ & 1 & 1 & 1 & 1 & 1 & 1 & 1 & 1 & 1 & 1 \\
\hline
\end{tabular}


Table 4 The values of the $E_{i j}^{*, \alpha} \mathrm{s}$ for different levels of $\alpha \in(0,1]$

\begin{tabular}{|c|c|c|c|c|c|c|c|c|c|c|}
\hline \multirow[t]{2}{*}{ Arcs } & \multicolumn{10}{|l|}{$E_{i j}^{*, \alpha}$} \\
\hline & $\alpha=0.1$ & $\alpha=0.2$ & $\alpha=0.3$ & $\alpha=0.4$ & $\alpha=0.5$ & $\alpha=0.6$ & $\alpha=0.7$ & $\alpha=0.8$ & $\alpha=0.9$ & $\overline{\alpha=1}$ \\
\hline $1 \longrightarrow 2$ & 1 & 1 & 1 & 1 & 0.98 & 0.95 & 0.93 & 0.9 & 0.88 & 0.86 \\
\hline $1 \longrightarrow 3$ & 0.96 & 0.89 & 0.78 & 0.66 & 0.56 & 0.47 & 0.4 & 0.36 & 0.31 & 0.28 \\
\hline $1 \longrightarrow 4$ & 1 & 0.98 & 0.96 & 0.95 & 0.93 & 0.91 & 0.9 & 0.88 & 0.87 & 0.85 \\
\hline $2 \longrightarrow 3$ & 1 & 1 & 1 & 1 & 1 & 1 & 1 & 1 & 1 & 1 \\
\hline $2 \longrightarrow 4$ & 1 & 1 & 1 & 1 & 1 & 1 & 1 & 1 & 1 & 0.99 \\
\hline $2 \longrightarrow 5$ & 0.92 & 0.91 & 0.9 & 0.9 & 0.89 & 0.88 & 0.87 & 0.87 & 0.86 & 0.85 \\
\hline $3 \longrightarrow 4$ & 1 & 1 & 1 & 1 & 1 & 0.99 & 0.98 & 0.97 & 0.96 & 0.95 \\
\hline $3 \longrightarrow 5$ & 0.39 & 0.38 & 0.37 & 0.36 & 0.36 & 0.35 & 0.34 & 0.33 & 0.33 & 0.32 \\
\hline $3 \longrightarrow 6$ & 0.93 & 0.92 & 0.91 & 0.9 & 0.89 & 0.88 & 0.87 & 0.86 & 0.85 & 0.84 \\
\hline $4 \longrightarrow 5$ & 1 & 1 & 1 & 1 & 1 & 1 & 1 & 1 & 1 & 1 \\
\hline $4 \longrightarrow 6$ & 0.43 & 0.42 & 0.41 & 0.39 & 0.38 & 0.37 & 0.36 & 0.36 & 0.35 & 0.34 \\
\hline $5 \longrightarrow 6$ & 1 & 1 & 1 & 1 & 1 & 1 & 1 & 1 & 1 & 1 \\
\hline $5 \longrightarrow 7$ & 1 & 1 & 1 & 1 & 1 & 1 & 1 & 1 & 1 & 1 \\
\hline $5 \longrightarrow 8$ & 0.72 & 0.72 & 0.71 & 0.71 & 0.71 & 0.7 & 0.7 & 0.69 & 0.69 & 0.69 \\
\hline $6 \longrightarrow 7$ & 0.73 & 0.72 & 0.72 & 0.72 & 0.72 & 0.72 & 0.71 & 0.7 & 0.7 & 0.69 \\
\hline $6 \longrightarrow 8$ & 1 & 1 & 1 & 1 & 1 & 1 & 1 & 1 & 1 & 1 \\
\hline $7 \longrightarrow 8$ & 1 & 1 & 1 & 1 & 1 & 1 & 1 & 1 & 1 & 1 \\
\hline
\end{tabular}

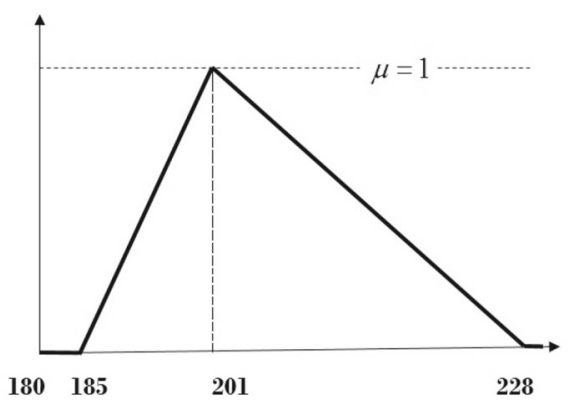

(a) Membership function of input 1 .

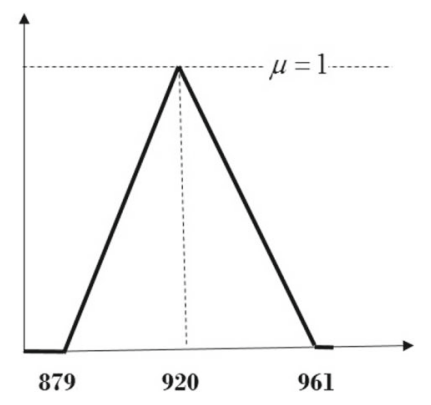

(b) Membership function of input 2 .

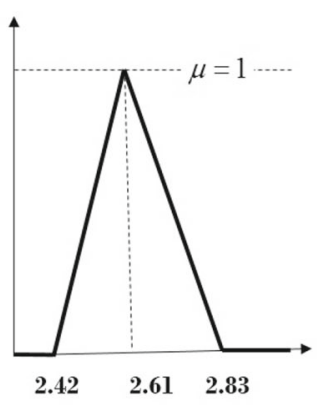

(c) Membership function of output 1 .

Fig. 3 The membership function of the objective functions for $\alpha=0.1$

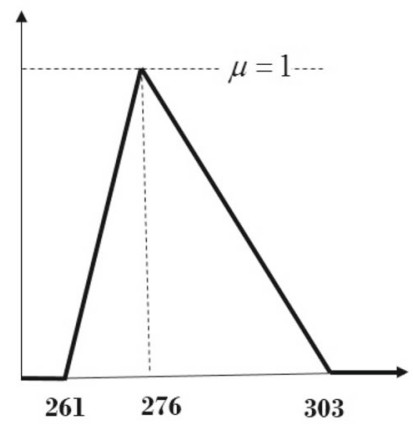

(a) Membership function of input 1 .
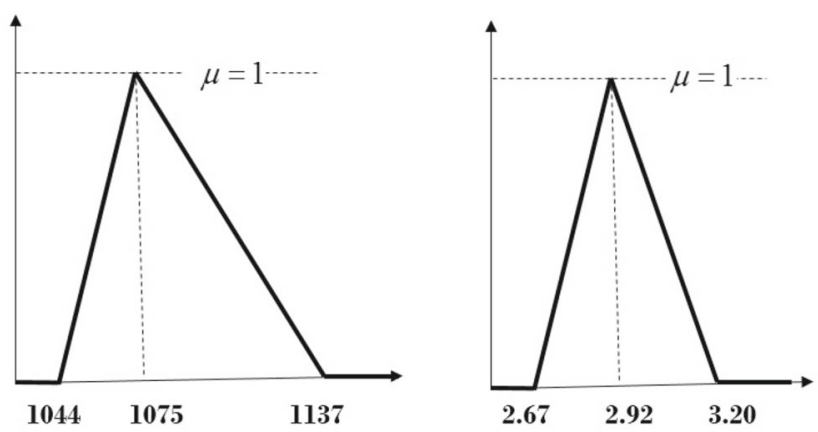

(b) Membership function of input 2. (c) Membership function of output 1.

Fig. 4 The membership function of the objective functions for $\alpha=0.2=0.3=\ldots=0.9=1$ 


$$
\begin{aligned}
& \sum_{(i, j) \in A} \tilde{c}_{i j}^{t} x_{i j} \leq \tilde{p}_{t}+\tilde{f}_{t}^{*}, \quad t=1,2, \ldots, k, \\
& \sum_{(i, j) \in A} \tilde{c}_{i j}^{t} x_{i j}+\tilde{n}_{t} \geq \tilde{f}_{t}^{*}, \quad t=k+1,2, \ldots, k+s, \\
& \tilde{p}_{t} \geq \tilde{0}, \quad t=1,2, \ldots, k, \\
& \tilde{n}_{t} \geq \tilde{0}, \quad t=k+1,2, \ldots, k+s, \\
& \sum_{j:(1, j) \in A} x_{i j}-\sum_{l:(l, 1) \in A} x_{l 1}=1, \\
& \sum_{j:(i, j) \in A} x_{i j}-\sum_{l:(l, i) \in A} x_{l i}=1, \text { for all }(i, j) \in A, i \neq 1, n, \\
& \sum_{j:(n, j) \in A} x_{i j}-\sum_{l:(l, n) \in A} x_{l 1}=-1, \\
& x_{i j}=0,1, \quad \text { for all }(i, j) \in A .
\end{aligned}
$$

Note 6.1 Model (31) is a fuzzy linear programming problem that can be solved using the proposed approach by Ebrahimnejad [46]. This approach is based on the order suggested by Ramik and Rimanek [47] for the fuzzy shortest path problem as follows:

Theorem 6.2 Let $\tilde{A}=\left(a_{l}, a_{m}, a_{u}\right)$ and $\tilde{B}=\left(b_{l}, b_{m}, b_{u}\right)$ be two triangular fuzzy numbers. Then $\tilde{A} \preceq \tilde{B}$ if, and only if, $a_{l} \leq b_{l}, a_{m} \leq b_{m}, a_{u} \leq b_{u}$.

Note 6.3 For solving fuzzy problem (31) assume $\tilde{n}_{t}=$ $\left(n_{t}^{l}, n_{t}^{m}, n_{t}^{u}\right), \tilde{p}_{t}=\left(p_{t}^{l}, p_{t}^{m}, p_{t}^{u}\right), \tilde{c}_{i j}^{t}=\left(c_{i j}^{l}, c_{i j}^{m}, c_{i j}^{u}\right)$ and $\tilde{f}_{t}^{*}=\left(f_{t}^{* l}, f_{t}^{* m}, f_{t}^{* u}\right)$. In this case, the fuzzy problem $(31)$ is converted into the following model regarding Definition 2.8 and Theorem 6.2.

\begin{tabular}{|c|c|c|c|}
\hline Arcs & Fuzzy cost & Fuzzy time & Fuzzy profit \\
\hline $1 \longrightarrow 2$ & $(80,100,110)$ & $(30,40,50)$ & $(450,500,600)$ \\
\hline $1 \longrightarrow 3$ & $(250,300,820)$ & $(20,25,35)$ & $(600,650,700)$ \\
\hline $1 \longrightarrow 4$ & $(10,25,35)$ & $(10,20,30)$ & $(550,600,750)$ \\
\hline $2 \longrightarrow 3$ & $(135,150,200)$ & $(15,20,25)$ & $(470,500,550)$ \\
\hline $2 \longrightarrow 5$ & $(90,100,110)$ & $(12,15,18)$ & $(125,150,200)$ \\
\hline $2 \longrightarrow 6$ & $(180,220,240)$ & $(30,40,50)$ & $(800,900,950)$ \\
\hline $3 \longrightarrow 4$ & $(120,130,150)$ & $(20,25,35)$ & $(450,500,600)$ \\
\hline $3 \longrightarrow 5$ & $(100,150,180)$ & $(30,40,50)$ & $(250,280,300)$ \\
\hline $3 \longrightarrow 6$ & $(110,140,160)$ & $(30,45,55)$ & $(125,135,140)$ \\
\hline $3 \longrightarrow 7$ & $(300,400,450)$ & $(25,35,40)$ & $(725,750,800)$ \\
\hline $4 \longrightarrow 6$ & $(120,160,180)$ & $(10,20,30)$ & $(625,650,700)$ \\
\hline $4 \longrightarrow 7$ & $(150,200,230)$ & $(10,20,30)$ & $(100,150,180)$ \\
\hline $5 \longrightarrow 6$ & $(80,90,100)$ & $(8,12,15)$ & $(100,150,180)$ \\
\hline $5 \longrightarrow 8$ & $(200,300,350)$ & $(50,55,65)$ & $(135,145,160)$ \\
\hline $5 \longrightarrow 9$ & $(120,150,170)$ & $(10,20,30)$ & $(625,650,700)$ \\
\hline $6 \longrightarrow 7$ & $(210,230,240)$ & $(20,25,35)$ & $(175,190,220)$ \\
\hline $6 \longrightarrow 8$ & $(150,180,200)$ & $(70,75,80)$ & $(160,170,190)$ \\
\hline $6 \longrightarrow 9$ & $(100,130,150)$ & $(20,30,35)$ & $(190,220,250)$ \\
\hline $6 \longrightarrow 10$ & $(70,90,100)$ & $(15,20,25)$ & $(750,80,900)$ \\
\hline $7 \longrightarrow 9$ & $(60,70,80)$ & $(20,25,30)$ & $(230,270,300)$ \\
\hline $7 \longrightarrow 10$ & $(50,60,70)$ & $(40,45,55)$ & $(220,250,300)$ \\
\hline $8 \longrightarrow 9$ & $(120,140,160)$ & $(55,65,70)$ & $(110,140,180)$ \\
\hline $8 \longrightarrow 11$ & $(110,130,150)$ & $(20,30,35)$ & $(120,150,170)$ \\
\hline $8 \longrightarrow 12$ & $(40,60,70)$ & $(15,20,25)$ & $(215,230,260)$ \\
\hline $9 \longrightarrow 10$ & $(110,140,160)$ & $(20,25,30)$ & $(200,250,280)$ \\
\hline $9 \longrightarrow 11$ & $(180,210,230)$ & $(55,65,75)$ & $(140,160,200)$ \\
\hline $9 \longrightarrow 12$ & $(300,370,400)$ & $(50,60,65)$ & $(190,220,250)$ \\
\hline $9 \longrightarrow 13$ & $(70,90,100)$ & $(55,65,70)$ & $(340,380,400)$ \\
\hline $10 \longrightarrow 12$ & $(150,180,200)$ & $(50,60,80)$ & $(165,170,190)$ \\
\hline $10 \longrightarrow 13$ & $(100,140,160)$ & $(70,80,85)$ & $(220,250,300)$ \\
\hline $11 \longrightarrow 12$ & $(50,70,80)$ & $(80,90,100)$ & $(340,380,400)$ \\
\hline $11 \longrightarrow 14$ & $(270,300,350)$ & $(50,60,70)$ & $(110,140,180)$ \\
\hline $12 \longrightarrow 13$ & $(100,120,150)$ & $(60,80,90)$ & $(215,240,250)$ \\
\hline $12 \longrightarrow 14$ & $(100,120,150)$ & $(100,120,140)$ & $(135,150,180)$ \\
\hline $13 \longrightarrow 14$ & $(200,230,260)$ & $(25,35,40)$ & $(265,285,300)$ \\
\hline
\end{tabular}

$\min \sum_{t=1}^{k}\left(p_{t}^{l}+p_{t}^{m}+p_{t}^{u}\right)+\sum_{t=k+1}^{k+s}\left(n_{t}^{l}+n_{t}^{m}+n_{t}^{u}\right)$

s.t.

$$
\begin{aligned}
& \sum_{(i, j) \in A} c_{i j}^{t, l} x_{i j} \leq p_{t}^{l}+f_{t}^{* l}, \quad t=1,2, \ldots, k, \\
& \sum_{(i, j) \in A} c_{i j}^{t, m} x_{i j} \leq p_{t}^{m}+f_{t}^{* m}, \quad t=1,2, \ldots, k,
\end{aligned}
$$

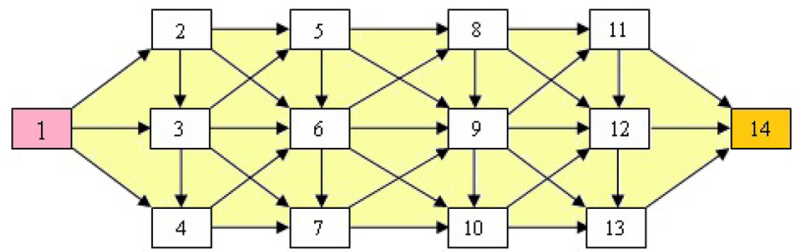

Fig. 5 A transportation network with 14 nodes and 35 arcs
Table 5 The input/output data of the transportation network for Example 5.2

$$
\begin{aligned}
& \sum_{(i, j) \in A} c_{i j}^{t, u} x_{i j} \leq p_{t}^{u}+f_{t}^{* u}, \quad t=1,2, \ldots, k, \\
& \sum_{(i, j) \in A} c_{i j}^{t, l} x_{i j}+n_{t}^{l} \geq f_{t}^{* l}, \quad t=k+1,2, \ldots, k+s, \\
& \sum_{(i, j) \in A} c_{i j}^{t, m} x_{i j}+n_{t}^{m} \geq f_{t}^{* m}, \quad t=k+1,2, \ldots, k+s, \\
& \sum_{(i, j) \in A} c_{i j}^{t, u} x_{i j}+n_{t}^{u} \geq f_{t}^{* u}, \quad t=k+1,2, \ldots, k+s, \\
& p_{t}^{l} \geq 0, p_{t}^{m} \geq p_{t}^{l}, p_{t}^{u} \geq p_{t}^{m} \quad t=1,2, \ldots, k,
\end{aligned}
$$


Table 6 The values of the $E_{i j}^{*, \alpha}$ s for different levels of $\alpha \in(0,1]$ in $G_{2}=\left(V_{2}, A_{2}\right)$

\begin{tabular}{|c|c|c|c|c|c|c|c|c|c|c|}
\hline Arcs & $\begin{array}{l}E_{i j}^{*, \alpha} \\
\alpha=0.1\end{array}$ & $\alpha=0.2$ & $\alpha=0.3$ & $\alpha=0.4$ & $\alpha=0.5$ & $\alpha=0.6$ & $\alpha=0.7$ & $\alpha=0.8$ & $\alpha=0.9$ & $\alpha=1$ \\
\hline $1 \longrightarrow 2$ & 0.99 & 0.95 & 0.91 & 0.87 & 0.84 & 0.81 & 0.78 & 0.75 & 0.73 & 0.71 \\
\hline $1 \longrightarrow 3$ & 1 & 1 & 1 & 1 & 1 & 1 & 1 & 1 & 0.96 & 0.92 \\
\hline $1 \longrightarrow 4$ & 1 & 1 & 1 & 1 & 1 & 1 & 1 & 1 & 1 & 1 \\
\hline $2 \longrightarrow 3$ & 1 & 1 & 1 & 1 & 1 & 1 & 1 & 1 & 1 & 1 \\
\hline $2 \longrightarrow 5$ & 0.92 & 0.89 & 0.86 & 0.84 & 0.81 & 0.79 & 0.77 & 0.75 & 0.73 & 0.71 \\
\hline $2 \longrightarrow 6$ & 1 & 1 & 1 & 1 & 1 & 1 & 1 & 1 & 1 & 1 \\
\hline $3 \longrightarrow 4$ & 1 & 1 & 1 & 1 & 1 & 0.98 & 0.94 & 0.9 & 0.86 & 0.83 \\
\hline $3 \longrightarrow 5$ & 0.96 & 0.93 & 0.9 & 0.87 & 0.84 & 0.82 & 0.8 & 0.78 & 0.76 & 0.74 \\
\hline $3 \longrightarrow 6$ & 0.38 & 0.36 & 0.34 & 0.32 & 0.31 & 0.29 & 0.28 & 0.27 & 0.25 & 0.24 \\
\hline $3 \longrightarrow 7$ & 1 & 1 & 1 & 1 & 1 & 1 & 1 & 1 & 1 & 1 \\
\hline $4 \longrightarrow 6$ & 1 & 1 & 1 & 1 & 1 & 1 & 1 & 1 & 1 & 1 \\
\hline $4 \longrightarrow 7$ & 0.8 & 0.71 & 0.62 & 0.55 & 0.49 & 0.44 & 0.41 & 0.37 & 0.34 & 0.31 \\
\hline $5 \longrightarrow 6$ & 0.97 & 0.88 & 0.79 & 0.71 & 0.65 & 0.58 & 0.53 & 0.47 & 0.43 & 0.4 \\
\hline $5 \longrightarrow 8$ & 0.6 & 0.59 & 0.59 & 0.58 & 0.58 & 0.57 & 0.57 & 0.56 & 0.56 & 0.56 \\
\hline $5 \longrightarrow 9$ & 1 & 1 & 1 & 1 & 1 & 1 & 1 & 1 & 1 & 1 \\
\hline $6 \longrightarrow 7$ & 0.48 & 0.46 & 0.44 & 0.42 & 0.4 & 0.38 & 0.36 & 0.35 & 0.33 & 0.31 \\
\hline $6 \longrightarrow 8$ & 0.58 & 0.58 & 0.57 & 0.57 & 0.57 & 0.56 & 0.56 & 0.56 & 0.56 & 0.55 \\
\hline $6 \longrightarrow 9$ & 0.51 & 0.48 & 0.45 & 0.42 & 0.39 & 0.37 & 0.35 & 0.32 & 0.31 & 0.29 \\
\hline $6 \longrightarrow 10$ & 1 & 1 & 1 & 1 & 1 & 1 & 1 & 1 & 1 & 1 \\
\hline $7 \longrightarrow 9$ & 1 & 1 & 1 & 1 & 1 & 1 & 1 & 0.98 & 0.96 & 0.94 \\
\hline $7 \longrightarrow 10$ & 0.88 & 0.86 & 0.84 & 0.82 & 0.81 & 0.79 & 0.77 & 0.76 & 0.75 & 0.73 \\
\hline $8 \longrightarrow 9$ & 0.42 & 0.4 & 0.38 & 0.35 & 0.33 & 0.31 & 0.3 & 0.28 & 0.26 & 0.25 \\
\hline $8 \longrightarrow 11$ & 0.95 & 0.91 & 0.88 & 0.85 & 0.82 & 0.8 & 0.77 & 0.75 & 0.73 & 0.72 \\
\hline $8 \longrightarrow 12$ & 1 & 1 & 1 & 1 & 1 & 1 & 1 & 1 & 1 & 1 \\
\hline $9 \longrightarrow 10$ & 0.72 & 0.71 & 0.69 & 0.68 & 0.67 & 0.66 & 0.65 & 0.64 & 0.63 & 0.62 \\
\hline $9 \longrightarrow 11$ & 0.8 & 0.78 & 0.76 & 0.74 & 0.7 & 0.66 & 0.61 & 0.57 & 0.53 & 0.49 \\
\hline $9 \longrightarrow 12$ & 0.62 & 0.58 & 0.54 & 0.5 & 0.47 & 0.44 & 0.41 & 0.39 & 0.36 & 0.34 \\
\hline $9 \longrightarrow 13$ & 1 & 1 & 1 & 1 & 1 & 1 & 1 & 1 & 1 & 1 \\
\hline $10 \longrightarrow 12$ & 0.71 & 0.7 & 0.69 & 0.68 & 0.67 & 0.66 & 0.65 & 0.63 & 0.6 & 0.57 \\
\hline $10 \longrightarrow 13$ & 0.92 & 0.9 & 0.88 & 0.86 & 0.84 & 0.83 & 0.81 & 0.8 & 0.78 & 0.77 \\
\hline $11 \longrightarrow 12$ & 1 & 1 & 1 & 1 & 1 & 1 & 1 & 1 & 1 & 1 \\
\hline $11 \longrightarrow 14$ & 0.8 & 0.75 & 0.7 & 0.66 & 0.62 & 0.59 & 0.55 & 0.52 & 0.49 & 0.46 \\
\hline $12 \longrightarrow 13$ & 0.91 & 0.88 & 0.86 & 0.85 & 0.83 & 0.81 & 0.8 & 0.78 & 0.77 & 0.76 \\
\hline $12 \longrightarrow 14$ & 1 & 1 & 1 & 0.98 & 0.94 & 0.91 & 0.89 & 0.86 & 0.83 & 0.81 \\
\hline $13 \longrightarrow 14$ & 1 & 1 & 1 & 1 & 1 & 1 & 1 & 1 & 1 & 1 \\
\hline
\end{tabular}

$n_{t}^{l} \geq 0, n_{t}^{m} \geq n_{t}^{l} n_{t}^{u} \geq n_{t}^{m}$

$t=k+1,2, \ldots, k+s$,

Constraints of Model (30).

Model (32) is a linear programming problem that can be solved using the simplex method.

It is worthwhile mentioning that both fuzzy DEA approach proposed in this study and the extended fuzzy GP approach reduces the FMOSPP (7) into a single objective problem. However, there are several important reasons for using our proposed method compared with the GP technique:
- The classical LP problem (32) applied for solving FMOSPP (7) is not a shortest path structured problem, whereas problem (19) for solving FMOSPP (7) are classical shortest path problem.

- The classical LP problem (32) applied for solving FMOSPP (7) has $9(k+s)+m$ constraints (without considering the non-negative constraints) and $3(k+s)+n$ variables (without considering slack variables), whereas problem (19) has only $m$ constraints and $n$ variables. Hence, from a computation point of view the proposed fuzzy DEA 
approach is preferable to the fuzzy GP technique for solving the FMOSPP (7).

- By use of the fuzzy DEA approach proposed in this study, the FMOSPP (7) is converted into a single objective fuzzy SPP keeping the structure of the SP problem, whereas using GP approach increases the number of constraints and variables of the problem by adding new constraints to the shortest path problem. Therefore, utilizing the proposed technique to is highly economical in comparison with the goal programming technique from a computational viewpoint, considering the number of constraints and variables.

- In the case that the problem requires an integer optimal solution, the fuzzy DEA approach can obtain an optimal solution to the SPP by ignoring the integrality restrictions, while fuzzy GP approach is not able to find integer solutions without adding the integrality restrictions.

Anyway, by solving model (30) corresponding to the FMOSPP (26), aspiration level vector is obtained as $\tilde{f}^{*}=$ $\left(\tilde{f}_{1}^{*}, \tilde{f}_{2}^{*}, \tilde{f}_{3}^{*}\right)=[(185,201,228),(573,610,645),(4.67$, $5.01,5.31)]$. Then, by solving the problem (32), the corresponding efficient path is obtained as $1 \rightarrow 4 \rightarrow 5 \rightarrow 8$. According to this path, the fuzzy value of Cost, Time and Safety are given as $(226,245,269),(573,610,645)$ and $(1.49,1.59,1.73)$, respectively. As can be seen, corresponding to this path, the fuzzy value of Time has reached the best possible value; however, it should be noted that the fuzzy value of Safety $(1.49,1.59,1.73)$ which is derived using model (32) is less than the both fuzzy values of Safety which is achieved using the proposed method shown in Figs. 3 and 4. Moreover, the fuzzy value of Cost $(226,245,269)$ which is derived using model (32) is greater than the fuzzy value of Cost $(185,201,228)$ achieved using the proposed method in Fig. 3. Therefore, it can be said, the proposed method is at least preferable considering the safety of the shortest path. Furthermore, although both optimal solutions obtained using the GP technique and the proposed method are Pareto optimal solutions, the efficiency scores of shortest paths obtained from the proposed method are more than the one derived from the GP technique. Finally, model (32) is an integer linear programming problem while model (19) is a linear programming problem. Solving an integer linear programming problem is harder than a linear programming problem.

Similarly, by solving model (30) corresponding to Example 5.2, aspiration level vector is obtained as $\tilde{f}^{*}=$ $\left(\tilde{f}_{1}^{*}, \tilde{f}_{2}^{*}, \tilde{f}_{3}^{*}\right)=[(420,545,635),(120,165,200),(3360$, $3675,4120)]$. In the following, by solving the problem (32), the corresponding efficient path for Example 5.2 is obtained as $1 \rightarrow 2 \rightarrow 3 \rightarrow 4 \rightarrow 6 \rightarrow 10 \rightarrow 13 \rightarrow 14$ that resulted in the fuzzy value of Cost, Time and Profit as $(825,1000,1160),(185,240,290)$ and $(3230,3485,3950)$, respectively. Again, although both optimal solutions obtained using the fuzzy GP technique and the proposed method are Pareto optimal solutions, the efficiency score of shortest path obtained from the proposed method is more than the one derived from the GP technique.

\section{Conclusions}

In this paper, a DEA-based approach was proposed to solve the SPP with multiple fuzzy attributes according to each arc called "FMOSPP". The idea behind the proposed approach was to convert the multiple fuzzy attributes into a unique fuzzy attribute. To this end, each arc in the given FMOSPP was considered as a DMU. Then those objective functions that need to be maximized were used to define the outputs of DMU and those ones that need to be minimized were used to define the inputs of DMU. In the following, using two fuzzy efficiency scores, the unique fuzzy relative efficiency was defined to each arc. By considering the unique efficiency as the only attribute, the FMOSPP was converted into a single objective FSPP that can be solved to determine a path with maximum efficiency as an efficient path.

It is worth mentioning that all of the multiple fuzzy attributes were considered as non-negative triangular fuzzy numbers. Accordingly, we used the proposed method by Saati et al. [35] to obtain the mentioned fuzzy efficiency scores based on the $\alpha$-cut approach. With this method, instead of facing a FSPP, a classic SPP with crisp parameters would be obtained for any selected $\alpha \in(0,1]$ that can be solved using the standard algorithms for solving the SPP such as Dijkstra's algorithm [48]. One of the main advantages of the proposed fuzzy DEA approach relies on its computational simplicity, allowing for its implementation within complex network structures. In addition, a simple technique to solve a fuzzy multi objective shortest path problem would be to optimize the fuzzy utility function of decision maker. However, the mathematical representation of the fuzzy utility function may not be determined easily in many problems. But, the fuzzy DEA approach proposed in this paper provided an efficient plan for the FMOSPP without explicit the knowledge of the decision maker's fuzzy utility function. In sum, the proposed fuzzy DEA approach applied in this study not only gives the efficient path without any knowledge of decision-maker, but also keeps the structure of the shortest path problem.

At the end, it should be noted that this study links the fuzzy MOSPP and fuzzy DEA together. As a future research it would be worthwhile to solve the stochastic multi-objective capacitated transportation problem [49] via stochastic DEA approaches. As another research, someone may be interested in establishing links between MOSPP and DEA in the case that the arc weights are represented in terms of intuitionistic fuzzy numbers [50-52] and single-valued neutrosophic num- 
bers [53]. Moreover, the development of the proposed method for solving multi-objective all-pairs shortest path problem in an interval-valued fuzzy network [54] and an interval-valued Pythagorean fuzzy network [55] is left to the next study. Finally, the generalization of the proposed method for determining the efficient solution of multi-objective constrained shortest pat problem using new forms of fuzzy inference systems such as type-1 fuzzy logic systems [56,57], interval type-2 fuzzy logic systems [58] and generalized type-2 fuzzy logic system [57] is an interesting topic for future research.

Acknowledgements The authors would like to thank the anonymous reviewers and the associate editor for their insightful comments and suggestions.

Open Access This article is licensed under a Creative Commons Attribution 4.0 International License, which permits use, sharing, adaptation, distribution and reproduction in any medium or format, as long as you give appropriate credit to the original author(s) and the source, provide a link to the Creative Commons licence, and indicate if changes were made. The images or other third party material in this article are included in the article's Creative Commons licence, unless indicated otherwise in a credit line to the material. If material is not included in the article's Creative Commons licence and your intended use is not permitted by statutory regulation or exceeds the permitted use, you will need to obtain permission directly from the copyright holder. To view a copy of this licence, visit http://creativecomm ons.org/licenses/by/4.0/.

\section{References}

1. Sastry VN, Janakiraman TN, Mohideen SI (2003) New algorithms for multi objective shortest path problem. Opsearch 40(4):278-298

2. Martins E, Santos J (1999) The labeling algorithm for the multiobjective shortest path problem, Departamento de Matematica, Universidade de Coimbra. TR-99/005, Portugal

3. Sauvanet G, Neron E (2010) Search for the best compromise solution on multiobjective shortest path problem. Electron Notes Discr Math 36:615-622

4. Pulido FJ, Mandow L, de la Cruz JLP (2014) Multiobjective shortest path problems with lexicographic goal-based preferences. Eur J Oper Res 239(1):89-101

5. Shi N, Zhou S, Wang F, Tao Y, Liu L (2017) The multi-criteria constrained shortest path problem. Transp Res Part E Log Transp Rev 101:13-29

6. Cintrano C, Chicano F, Alba E (2019) Facing robustness as a multiobjective problem: a bi-objective shortest path problem in smart regions. Inf Sci 503:255-273

7. Zajac S, Huber S (2020) Objectives and methods in multi-objective routing problems: a survey and classification scheme. Eur J Oper Res

8. Ajeil FH, Ibraheem IK, Sahib MA, Humaidi AJ (2020) Multiobjective path planning of an autonomous mobile robot using hybrid PSO-MFB optimization algorithm. Appl Soft Comput 89:106076

9. Dehghani M, Vahdat V, Amiri M, Rabiei E, Salehi S (2019) A multi-objective optimization model for a reliable generalized flow network design. Comput Ind Eng 138:106074

10. Cooper WW, Seiford LM, Tone K (2006) Introduction to data envelopment analysis and its uses: with DEA-solver software and references. Springer, New York
11. Masoumi MM, Lotfi FH, Mobasseri AM (2010) DEA and multiobjective shortest path problems. Contemp Eng Sci 3(2):53-62

12. Amirteimoori A (2012) An extended shortest path problem: a data envelopment analysis approach. Appl Math Lett 25(11):1839-1843

13. Rani GV, Reddy B (2017) Multi-objective fuzzy shortest path selection for green routing and scheduling problems. International Journal of Advanced Research in Computer Science 8(7):47-475

14. Zero L, Bersani C, Paolucci M, Sacile R (2019) Two new approaches for the bi-objective shortest path with a fuzzy objective applied to HAZMAT transportation. J Hazard Mater 375:96-106

15. Hasuike $T$ (2013) Robust shortest path problem based on a confidence interval in fuzzy bicriteria decision making. Inf Sci 221:520-533

16. Patle BK, Jha A, Pandey A, Gudadhe N, Kashyap SK (2019) The optimized path for a mobile robot using fuzzy decision function. Mater Today Proc 18:3575-3581

17. Abbaszadeh Sori A, Ebrahimnejad A, Motameni H (2020) The fuzzy inference approach to solve multi-objective constrained shortest path problem. J Intell Fuzzy Syst 32:4711-4720

18. Majumder S, Kar MB, Kar S, Pal T (2020) The fuzzy inference approach to solve multi-objective constrained shortest path problem. Soft Comput 24:8975-8996

19. Deng Y, Chen Y, Zhang Y, Mahadevan S (2012) Fuzzy Dijkstra algorithm for shortest path problem under uncertain environment. Appl Soft Comput 12(3):1231-1237

20. Ebrahimnejad A, Tavana M, Alrezaamiri H (2016) A novel artificial bee colony algorithm for shortest path problems with fuzzy arc weights. Measurement 93:48-56

21. Tajdin A, Mahdavi I, Mahdavi-Amiri N, Sadeghpour-Gildeh B (2010) Computing a fuzzy shortest path in a network with mixed fuzzy arc lengths using $\alpha$-cuts. Comput Math Appl 60(4):989-1002

22. Dudeja C (2019) Fuzzy-based modified particle swarm optimization algorithm for shortest path problems. Soft Comput 23:83218331

23. Abbaszadeh Sori A, Ebrahimnejad A, Motameni H (2020) Elite artificial bees' colony algorithm to solve robot's fuzzy constrained routing problem. Comput Intell 36:659-681

24. Broumi S, Talea M, Bakali A, Smarandache F, Nagarajan D, Lathamaheswari M, Parimala M (2019) Shortest path problem in fuzzy, intuitionistic fuzzy and neutrosophic environment: an overview. Complex Intell Syst 5:371-378

25. Sengupta JK (1992) A fuzzy systems approach in data envelopment analysis. Comput Math Appl 24(8-9):259-266

26. Chen CT (2001) A fuzzy approach to select the location of the distribution center. Fuzzy Sets Syst 118(1):65-73

27. Saati S, Memariani A (2009) SBM model with fuzzy input-output levels in DEA. Aust J Basic Appl Sci 3(2):352-357

28. Hatami-Marbini A, Saati S (2009) Stability of RTS of efficient DMUs in DEA with fuzzy. Appl Math Sci 3(44):2157-2166

29. Asady B, Zendehnam A (2007) Ranking fuzzy numbers by distance minimization. Appl Math Model 11:2589-2598

30. Sheth N, Triantis K (2003) Measuring and evaluating efficiency and effectiveness using goal programming and data envelopment analysis in a fuzzy environment. Yugoslav J Oper Res 13(1):35-60

31. Azar A, Zarei Mahmoudabadi M, Emrouznejad A (2016) A new fuzzy additive model for determining the common set of weights in data envelopment analysis. J Intell Fuzzy Syst 30(1):61-69

32. Ebrahimi B, Dellnitz A, Kleine A, Tavana M (2021) A novel method for solving data envelopment analysis problems with weak ordinal data using robust measures. Expert Syst Appl 164:113835

33. Heydari C, Omrani H, Taghizadeh R (2020) A fully fuzzy network DEA-range adjusted measure model for evaluating airlines efficiency: a case of Iran. J Air Transp Manag 89:101923

34. Arana-Jimenez M, Sanchez-Gil M C, Lozano S (2020) A fuzzy DEA slacks-based approach. J Comput Appl Math 113180

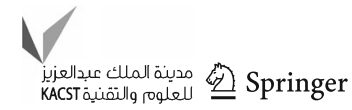


35. Saati SM, Memariani A, Jahanshahloo GR (2002) Efficiency analysis and ranking of DMUs with fuzzy data. Fuzzy Optim Decis Making 1(3):255-267

36. Charnes A, Cooper WW, Rhodes E (1978) Measuring the efficiency of decision making units. Eur J Oper Res 2(6):429-444

37. Ebrahimnejad A, Verdegay JL (2018) Fuzzy sets-based methods and techniques for modern analytics. Springer, New York

38. Ebrahimnejad A, Nasseri H, Gholami O (2019) Fuzzy stochastic data envelopment analysis with application to NATO enlargement problem. RAIRO Oper Res 53:705-721

39. Ebrahimnejad A, Verdegay JL, Garg H (2019) Signed distance ranking based approach for solving bounded intervalvalued fuzzy numbers linear programming problems. Int J Intell Syst 9(34):2055-2076

40. Hatami-Marbini A (2019) Benchmarking with network dea in a fuzzy environment. RAIRO Oper Res 53:687-703

41. Bai X, Zhang F, Liu Y (2018) Modeling fuzzy data envelopment analysis under robust input and output data. RAIRO Oper Res 53:619-643

42. Zadeh LA (1965) Fuzzy sets. Inf Control 8(3):338-353

43. Wang TC, Chen LY, Chen YH (2008, October) Applying fuzzy PROMETHEE method for evaluating IS outsourcing suppliers. In: 2008 Fifth International Conference on Fuzzy Systems and Knowledge Discovery (Vol. 3, pp. 361-365). IEEE

44. Ebrahimnejad A (2019) An effective computational attempt for solving fully fuzzy linear programming using MOLP problem. J Ind Product Eng 36(2):59-69

45. Zhou P, Ang BW, Poh KL (2008) A survey of data envelopment analysis in energy and environmental studies. Eur J Oper Res 189(1): $1-18$

46. Ebrahimnejad A (2016) New method for solving fuzzy transportation problems with LR flat fuzzy numbers. Inf Sci 357:108-124

47. Ramic J, Rimanek J (1985) Inequality relation between fuzzy numbers and its use in fuzzy optimization. Fuzzy Sets Syst 16(2):123-138

48. Dijkstra EW (1959) A note on two problems in connexion with graphs. Numer Math 1(1):269-271

49. Gupta S, Garg H, Chaudhary S (2020) Parameter estimation and optimization of multi-objective capacitated stochastic transportation problem for gamma distribution. Complex Intell Syst. https:// doi.org/10.1007/s40747-020-00156-1

50. Garg H, Singh S (2020) Algorithm for solving group decisionmaking problems based on the similarity measures under type 2 intuitionistic fuzzy sets environment. Soft Comput 24:7361-7381
51. Garg H (2020) Exponential operational laws and new aggregation operators for intuitionistic multiplicative set in multiple-attribute group decision making process. Inf Sci 538:245-272

52. Garg H (2020) New ranking method for normal intuitionistic sets under crisp, interval environments and its applications to multiple attribute decision making process. Complex Intell Syst. https://doi. org/10.1007/s40747-020-00150-7

53. Garg H (2020) Novel neutrality aggregation operator-based multiattribute group decision-making method for single-valued neutrosophic numbers. Soft Comput 24(14):10327-10349

54. Enayattabar M, Ebrahimnejad A, Motameni H (2019) Dijkstra algorithm for shortest path problem under interval-valued Pythagorean fuzzy environment. Complex Intell Syst 5(2):93-100

55. Enayattabar M, Ebrahimnejad A, Motameni H, Garg H (2019) A novel approach for solving all-pairs shortest path problem in an interval-valued fuzzy network .J Intell Fuzzy Syst 37:6865-6877

56. Castillo O, Cervantes L, Soria J, Sanchez M, Castro JR (2016) A generalized type-2 fuzzy granular approach with applications to aerospace. Inf Sci 354:165-177

57. Castillo O, Amador-Angulo L, Castro JR, Garcia-Valdez M (2016) A comparative study of type-1 fuzzy logic systems, interval type-2 fuzzy logic systems and generalized type-2 fuzzy logic systems in control problems. Inf Sci 354:257-274

58. Ontiveros-Robles E, Melin P, Castillo O (2018) Comparative analysis of noise robustness of type 2 fuzzy logic controllers. Kybernetika 54(1):175-201

Publisher's Note Springer Nature remains neutral with regard to jurisdictional claims in published maps and institutional affiliations. 